\title{
Influence of non-stationary electromagnetic field conditions on ion pick-up at Titan: 3-D multispecies hybrid simulations
}

\author{
S. Simon ${ }^{1}$, U. Motschmann ${ }^{1,2}$, and K.-H. Glassmeier ${ }^{3}$ \\ ${ }^{1}$ Institute for Theoretical Physics, TU Braunschweig, Germany \\ ${ }^{2}$ Institute for Planetary Research, DLR, Berlin, Germany \\ ${ }^{3}$ Institute for Geophysics and Extraterrestrial Physics, TU Braunschweig, Germany
}

Received: 26 August 2007 - Revised: 9 January 2008 - Accepted: 14 January 2008 - Published: 26 March 2008

\begin{abstract}
Titan's interaction with the corotating Saturnian magnetospheric plasma is studied in terms of a threedimensional electromagnetic hybrid model. This approach treats the electrons as a massless, charge-neutralizing fluid, whereas the ions are represented by macroparticles. The model considers two magnetospheric and three ionospheric ion species. In contrast to any foregoing simulation study, the magnetospheric upstream conditions are not assumed to be stationary, but time variations have been imposed on the electromagnetic fields. The model includes simple periodic distortions of the fields near Titan, the purpose being to illustrate the basic physical mechanisms of ion pick-up in a non-stationary electromagnetic environment. In order to allow a straightforward access to the influence of the electromagnetic field orientation on the pick-up, no variations have been imposed on the density of the impinging magnetospheric plasma. Under stationary upstream conditions, Titan's exospheric tail exhibits a strong asymmetry with respect to the direction of the convective electric field. The simulations show that this characteristic asymmetry cannot develop, if the ambient electromagnetic fields are highly distorted. However, the central tail region directly behind the satellite remains nearly unaffected by the distorted magnetospheric upstream conditions. The central tail where the slow ionospheric species are predominant is able to shield itself against any kind of distortion in the ambient magnetospheric field conditions. The shorter the time period of the distortions, the more efficient is this shielding effect. The dependency of the pick-up on the characteristic time scales of the distortions is discussed in detail for the investigated model cases. Besides, the reaction of Titan's exospheric tail structure on sudden, non-continuous changes of the magnetospheric plasma conditions is analyzed, providing an illus-
\end{abstract}

Correspondence to: S. Simon

(sven.simon@tu-bs.de) tration of some effects that may occur when Titan crosses Saturn's magnetopause.

Keywords. Magnetospheric physics (Magnetosphere interactions with satellites and rings; Magnetosphere-ionosphere interactions) - Space plasma physics (Kinetic and MHD theories; Numerical simulation studies)

\section{Introduction}

During the ongoing Cassini mission to the Saturnian system, more than 30 flybys of the giant planet's largest moon, Titan, have already been accomplished. The plasma and magnetic field data collected by the Cassini instruments (cf., for instance, Waite et al., 2005; Wahlund et al., 2005; Backes et al., 2005; Modolo et al., 2007b) have greatly improved our understanding of the interaction of Titan's dense, nitrogenrich atmosphere and ionosphere with the corotating Saturnian magnetospheric plasma flow. Numerical simulations provide an invaluable tool for the interpretation and analysis of the material obtained by the Cassini spacecraft. Recently, Ma et al. (2006) succeeded in reproducing some features of the magnetic field signatures detected during the first two Cassini flybys by means of a three-dimensional magnetohydrodynamic plasma model. This model has also been successfully applied to the magnetic field observations during the T9 encounter (Ma et al., 2007). Based on the magnetohydrodynamic plasma description, Neubauer et al. (2006) performed a comparative analysis of the magnetic field data collected during the Cassini TA, TB and T3 flybys. These authors demonstrate that some of the observed features can be understood by assuming the interaction to produce a set of Alfven wings in the vicinity of Titan. Recently, the first successful application of a multi-fluid model to Titan's plasma

Published by Copernicus Publications on behalf of the European Geosciences Union. 
interaction has been accepted for publication (Snowden et al., 2007).

However, despite its numerous advantages (cf. Simon et al., 2006b, 2007b, and references therein), the fluid plasma description is not strictly applicable to the interaction between Titan and Saturn's magnetospheric plasma flow. The gyroradii of newly generated ionospheric particles may exceed the diameter of the satellite by more than a factor of 5 . This has for instance been emphasized by Luhmann (1996) and by Ledvina et al. (2005). Therefore, an analysis of Titan's plasma interaction that includes a complete description of these effects requires to employ a semi-kinetic or hybrid simulation approach. While this approximation treats the electrons of the plasma as a massless, charge-neutralizing fluid, it is able to retain the kinetic character of ion dynamics. Large numbers of individual ions are merged to so-called macroparticles, whose dynamics are governed by the equation of motion known from classical electrodynamics:

$m \frac{\mathrm{d}^{2} \boldsymbol{x}}{\mathrm{d} t^{2}}=q\left(\boldsymbol{E}+\frac{\mathrm{d} \boldsymbol{x}}{\mathrm{d} t} \times \boldsymbol{B}\right)$.

In this expression, $\boldsymbol{E}$ and $\boldsymbol{B}$ are the electromagnetic fields that act on an ion of mass $m$ and charge $q$, its position being denoted by $\boldsymbol{x}$. The first who applied the hybrid approximation to the interaction between Titan and Saturn's magnetospheric plasma were Brecht et al. (2000). However, the authors neglected the finite temperature of the impinging magnetospheric plasma. Therefore, in the model, the interaction gave rise to a shock wave in front of the satellite. Nonetheless, the model yielded a correct description of the highly asymmetric structure of Titan's exospheric tail. Even though Kallio et al. (2004) were able to include the real subfast nature of the impinging magnetospheric plasma into their simulation, their hybrid model did not consider the finite pressure of the magnetospheric and ionospheric electrons. This problem has been overcome in the study of Simon et al. (2006b) who presented a systematic analysis of the structures in Titan's plasma environment as a function of the satellite's orbital position inside as well as outside the Saturnian magnetosphere. The strong analogy to the interaction of the solar wind with the Martian oxygen ionosphere has been emphasized in a subsequent comparative simulation study (Simon et al., 2007a).

However, most of the simulation models presented above do not consider the multi-species nature of Titan's plasma interaction. While the magnetohydrodynamic models typically include detailed and, due to the excellent grid resolution, highly sophisticated models of the ionosphere, plasma dynamic interactions are modeled by averaging all the ion species together into a single fluid with a single momentum equation. The hybrid models usually consider one magnetospheric species that features average properties as well as molecular nitrogen, being the most abundant heavy species of ionospheric origin. Within the framework of a multispecies hybrid model, Titan's plasma interaction has been studied by Simon et al. (2007b). The authors consider three different species of ionospheric origin. It has been demonstrated that since the gyroradii of the newly generated ionospheric ions depend linearly on their mass, different species become spatially dispersed in Titan's wake region. Besides, it has been shown by these authors that the pick-up processes of different ionospheric species cannot be understood independently of each other, but the pick-up of light ionospheric species is suppressed by the heavier ones. Simon et al. (2007b) also took account for the fact that the impinging magnetospheric plasma consists of two components with significantly different masses. Consequentially, their flow patterns in the vicinity of Titan are modified on different length scales. The authors refer to this as the magnetospheric mass spectrometer effect. Recently, this five-species model has been successfully applied to reproduce and explain some features of Titan's magnetic field signature during the ninth Cassini encounter (T9) on 26 December 2005 (Simon et al., 2007). The model has been applied to the ramside flyby T34, as well (Simon et al., 2008). A very similar model has been successfully applied to Titan's plasma interaction by Modolo et al. (2007a).

All simulation studies described above are based on the assumption of spatially homogeneous magnetospheric upstream conditions in the vicinity of Titan. Time variations in the upstream electromagnetic field and plasma parameters have been neglected, as well. Luhmann (1996) who studied the structure of Titan's exospheric tail in the framework of a test particle model suggested that short-scale magnetic fluctuations may have significant impact on the satellite's asymmetric tail structure, especially on the spatial dispersion of species of different masses (ionospheric mass spectrometer effect). This aspect has so far not been addressed by any of the global, three-dimensional simulation approaches. Most likely, the reason for this are the considerable numerical difficulties that go along with the incorporation of any kind of fluctuating upstream condition into a numerical simulation model. The present paper describes the results of the first attempt to include time variations in the upstream conditions of a three-dimensional hybrid model of Titan's plasma interaction.

As demonstrated in our companion papers, Titan's plasma tail exhibits a strong asymmetry with respect to the direction of the undisturbed convective electric field $\boldsymbol{E}_{c}$. The ionospheric tail is unable to penetrate into the hemisphere where $\boldsymbol{E}_{c}$ is directed towards Titan. In contrast to this, in the hemisphere where $\boldsymbol{E}_{c}$ points away from Titan, the ionospheric particles are being dragged away from the satellite. They gyrate on cycloidal pick-up trajectories, their extension in the direction of the electric field depending on the mass of the respective species. One major purpose of the present study is to understand how this tail structure is modified, if the ambient magnetospheric electric field does not point in the same direction during the entire simulation, but its orientation is continuously changed. 
Of course, due to the necessity to keep the simulation numerically stable, the field distortion included in the simulation model is somewhat artificial. It does not correspond to a real existing magnetospheric wave mode, especially not in the linear regime. However, the development of a planet's or moon's pick-up tail under the influence of varying electromagnetic field conditions is of general interest, not only for the case of Titan, but also for improving our understanding of the interaction between the Martian or a cometary ionosphere and the solar wind. Since it has already been well described in previous simulation studies, the Titan scenario can be considered a "plasma laboratory" in which some aspects of ion pick-up under varying conditions can be studied. Although this first simulation study of ion pick-up in a non-stationary electromagnetic environment does not claim to provide a quantitative reproduction of data collected by the Cassini plasma instruments, the results may be of value for our understanding of the observations during past and future Titan flybys. As already stated by Neubauer et al. (2006), ambient magnetospheric plasma conditions during the first series of Cassini flybys have not been perfectly undistorted, as it is so far assumed by any existing simulation model. As recently shown by Gurnett et al. (2007), the magnetic fields inside Saturn's magnetosphere exhibit a modulation with a period of about $10.7 \mathrm{~h}$. Our model provides first qualitative insights of how such periodic field distortions may affect the pick-up effect at Titan. As a hybrid simulation code operates on the ion gyroscales (i.e. on time scales of seconds or minutes), it is not possible to resolve the observed $10.7 \mathrm{~h}$ period with any existing computer. However, we can provide at least some basic conclusions of how a short-scale distortion would affect the satellite's tail structure.

The case of Titan crossing Saturn's magnetopause has recently been observed for the first time during the T32 flyby on 13 June 2007 (C. Bertucci et al., A. J. Coates et al., presentation of first results at AGU fall meeting 2007). During this flyby, a nearly complete reversal of the magnetic field was observed. Such a scenario is studied within our paper as well. Our simulation model presents a first attempt towards understanding the physical processes that take place in such a unique situation. Based on the existing models that use stationary upstream conditions, it is illustrated how this -well understood- situation is modified by sudden changes in the magnetic field, while the flow parameters themselves are not altered. Of course, our future work will focus on including the anticipated density changes of a magnetopause crossing as well. However, the behavior of the plasma moments, especially of the flow direction and composition during T32, is still unknown. Besides, as discussed in detail in our companion papers, according to our model, the shape and orientation of Titan's exospheric tail are almost completely determined by the direction of the undisturbed electromagnetic fields upstream of Titan, while the specific parameters of the magnetospheric plasma constituents play only a minor role. A similar decoupling of exospheric and impinging plasma flow dynamics has also been observed in our simulations of cometary plasma interactions, cf. Bagdonat and Motschmann (2001, 2002a,b). Therefore, although the distortions of the magnetospheric plasma components are not described fully realistic, the model should allow qualitative insights into the effect on the moon's exospheric wake.

This paper is organized as follows: in Sect. 2, we give an overview of the basic input parameters and the different kinds of distortions that have been imposed on the upstream electromagnetic fields. As stated above, the study does not only consider continuous changes in the ambient fields, but the influence of sudden changes (discontinuities) in the direction of the pick-up fields is discussed as well. In Sect. 3, we give a brief description of how Titan's ionospheric tail is shaped under stationary upstream conditions. Since this situation has been analyzed extensively in the companion paper by Simon et al. (2007b), we restrict the discussion to pointing out the key features of the tail structure. This brief summary mainly provides a reference for the interpretation of the new simulation results presented in Sects. 4 to 7, where time variations in the ambient electromagnetic fields are considered. While Sects. 4 to 6 refer to a continuous change in the direction of the pick-up fields, Sect. 7 describes how Titan's ionospheric tail structure is affected by the impact of tangential discontinuities. In order to gain good insight into the involved physics, two "extreme" situations are considered: rotations of the electromagnetic fields by 90 degrees and by 180 degrees, i.e. a complete reversal in the direction of the pick-up fields. The final section gives a short summary of our major results.

\section{Simulation model and input parameters}

The hybrid approach treats the electrons of the plasma as a massless, charge-neutralizing fluid, while the ions are represented by individual particles. A detailed description of the hybrid model used for this study has been given in our companion papers on Titan's plasma interaction mentioned above as well as in the thesis of Simon (2007). These papers also give a detailed discussion of how the electron populations that correspond to different ion species are handled. The electron parameters are exactly the same as in the paper by Simon et al. (2007b). The code used for this study has also been successfully applied to the interaction of weakly outgassing comets with the solar wind (Bagdonat and Motschmann, 2001, 2002a,b; Bagdonat, 2005; Motschmann and Kührt, 2006), to the plasma environment of Mars (Bößwetter et al., 2004) and to the interaction between a magnetized asteroid and the solar wind (Simon et al., 2006a), providing ancillary material for the Rosetta mission. As will be discussed below, the simulation model considers five different ion species.

The basic equations of the hybrid model can be written as follows: 


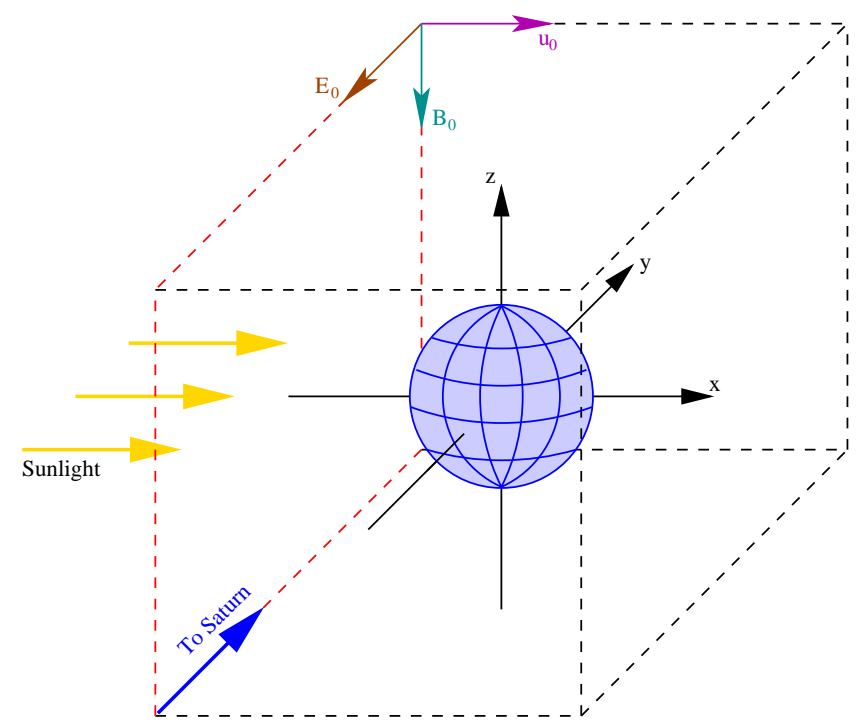

Fig. 1. Simulation geometry. The center of Titan coincides with the center of the cubic simulation domain, its extension being $\pm 7.5 R_{T}$ in each spatial direction. The upstream plasma direction $\boldsymbol{u}_{0}$ as well as the direction of the ionizing solar UV radiation are aligned with the positive $\mathrm{x}$-axis. In the case of stationary upstream conditions, the undisturbed magnetic field $\boldsymbol{B}_{0}$ is oriented antiparallel to the z-axis. This yields a convective electric field $\boldsymbol{E}_{0}=-\boldsymbol{u}_{0} \times \boldsymbol{B}_{0}$ that points in $(-y)$ direction, i.e. away from Saturn. All faces of the simulation box except for the left one (as denoted by the red dashed lines) have been treated as outflow boundaries. The left-hand face of the box has not simply been treated as an inflow boundary layer, but the electromagnetic fields in this face of the box have been varied in time during the simulations, the purpose being to mimic distorted magnetospheric upstream conditions in the vicinity of Titan. Details are discussed in Sect. 2 of the text.

- Equations of motion for individual ions:

$$
\frac{\mathrm{d} \boldsymbol{x}_{s}}{\mathrm{~d} t}=\boldsymbol{v}_{s} \quad \text { and } \quad \frac{\mathrm{d} \boldsymbol{v}_{s}}{\mathrm{~d} t}=\frac{q_{s}}{m_{s}}\left\{\boldsymbol{E}+\boldsymbol{v}_{s} \times \boldsymbol{B}\right\},
$$

where $\boldsymbol{x}_{s}$ and $\boldsymbol{v}_{s}$ denote the position and the velocity of an ion of species $s$, respectively. The vectors $\boldsymbol{E}$ and $\boldsymbol{B}$ are the electromagnetic fields. The ion mass and charge are denoted by $m_{s}$ and $q_{s}$, respectively.

- Electric field equation:

$$
\boldsymbol{E}=-\boldsymbol{u}_{i} \times \boldsymbol{B}+\frac{(\nabla \times \boldsymbol{B}) \times \boldsymbol{B}}{\mu_{0} e n_{e}}-\frac{\nabla P_{e, 1}+\nabla P_{e, 2}}{e n_{e}}
$$

where $\boldsymbol{u}_{i}$ is the mean ion velocity. The plasma is quasineutral, i.e. the mean ion $\left(n_{i}\right)$ and electron density $\left(n_{e}\right)$ are assumed to be equal. As in general, the electron temperature in a planetary ionosphere differs significantly from the electron temperature in the ambient plasma flow, two different electron pressure terms $P_{e, 1}$ and $P_{e, 2}$ have been incorporated into the simulation model. Both electron populations are described by adiabatic laws:

$P_{e, 1} \propto \beta_{e, 1} n_{e, 1}^{\kappa} \quad$ and $\quad P_{e, 2} \propto \beta_{e, 2} n_{e, 2}^{\kappa}$,

where $\beta$ are the plasma betas and $\kappa$ is the adiabatic exponent. For the simulations presented in this work, a value of $\kappa=2$ has been chosen (Bößwetter et al., 2004; Simon et al., 2006a,b).

- Magnetic field equation: By using Faraday's law, an expression describing the time evolution of the magnetic field can be obtained:

$$
\frac{\partial \boldsymbol{B}}{\partial t}=\nabla \times\left(\boldsymbol{u}_{i} \times \boldsymbol{B}\right)-\nabla \times\left[\frac{(\nabla \times \boldsymbol{B}) \times \boldsymbol{B}}{\mu_{0} e n_{e}}\right] .
$$

Because of the adiabatic description of the electrons, the electron pressure terms do not appear in this equation:

$$
\begin{aligned}
& \nabla \times\left(\frac{\nabla n_{e}^{\kappa}}{n_{e}}\right) \\
& =\frac{1}{n_{e}} \nabla \times\left[\nabla\left(n_{e}^{\kappa}\right)\right]+\left[\nabla \frac{1}{n_{e}}\right] \times\left[\nabla\left(n_{e}^{\kappa}\right)\right] \\
& =\left[\nabla \frac{1}{n_{e}}\right] \times\left[\nabla\left(n_{e}^{\kappa}\right)\right] \\
& =-\frac{1}{n_{e}^{2}}\left[\nabla n_{e}\right] \times \kappa n_{e}^{\kappa-1}\left[\nabla n_{e}\right] \\
& =0 .
\end{aligned}
$$

Due to its adequacy for illustrating basic plasma-physical processes, we consider the "classical" Voyager 1 geometry of Titan's interaction with the Saturnian magnetospheric plasma (cf. for instance Neubauer et al., 1984; Ledvina et al., 2004; Backes, 2005; Backes et al., 2005). The upstream flow is assumed to consist of two species of masses $m\left(\mathrm{O}^{+}\right)=16 \mathrm{amu}$ and $m\left(\mathrm{H}^{+}\right)=1 \mathrm{amu}$, their densities being $n\left(\mathrm{O}^{+}\right)=0.2 \times 10^{6} \mathrm{~m}^{-3}$ and $n\left(\mathrm{H}^{+}\right)=n\left(\mathrm{O}^{+}\right) / 2$. The upstream flow velocity is given by $\boldsymbol{u}_{0}=120 \mathrm{~km} / \mathrm{s} \boldsymbol{e}_{x}$, where $\boldsymbol{e}_{x}$ is the unit vector in positive $x$-direction (cf. Fig. 1). The undisturbed magnetic field is directed perpendicular to Titan's orbital plane and points in $(-z)$ direction, i.e. $\boldsymbol{B}_{0}=-5 \mathrm{nT} \boldsymbol{e}_{z}$. Thus, the undisturbed convective electric field

$\boldsymbol{E}_{0}=-\boldsymbol{u}_{0} \times \boldsymbol{B}_{0}=-0.6 \mathrm{~V} / \mathrm{km} \boldsymbol{e}_{y}$

is directed away from Saturn. The Mach numbers of the magnetospheric upstream flow are given by $M_{A}=1.87$ (alfvenic), $M_{S}=0.57$ (sonic) and $M_{M S}=0.55$ (magnetosonic). Titan's dayside ionosphere is located in the $(x<0)$ hemisphere, i.e. we refer to the situation at 18:00 local time at the equinoxes of Saturn's orbit around the sun. This geometry is described in detail in our previous papers. The ionosphere is assumed to consist of three species of representative masses: molecular nitrogen, methane and molecular hydrogen. Titan's dayside ionosphere is generated by solar UV radiation and is 
therefore represented by a Chapman layer. Particle impact processes at the nightside are approximated by a production profile that depends on the altitude above the surface, but not on the solar zenith angle. Details of the ionosphere model are described by Simon et al. (2006b). The simulations are carried out on a curvilinear simulation grid, allowing to achieve an enhanced spatial resolution near the ionospheric production maximum. The cubic simulation domain of extension $\left( \pm 7.5 R_{T}\right) \times\left( \pm 7.5 R_{T}\right) \times\left( \pm 7.5 R_{T}\right)$ (radius of Titan: $\left.R_{T}=2575 \mathrm{~km}\right)$ is discretized by 90 grid nodes in each direction. The specific curvature parameters of the grid are exactly the same as in our companion paper (Simon et al., 2006b). This work also contains an illustration of the grid, showing the locations of regions with high spatial resolution. We achieve a minimum resolution of about $250 \mathrm{~km}$. The inner boundary of the ionosphere is located at an altitude of $700 \mathrm{~km}$ above Titan's surface. The boundary conditions that are imposed on the plasma parameters and the vector fields (particle-absorbing sphere, no boundary conditions set for $\boldsymbol{E}$ and $\boldsymbol{B}$ ) are discussed in detail in our companion paper ( $\mathrm{Si}-$ mon et al., 2006b). The same boundary conditions have also been successfully applied to our hybrid model of the Martian plasma interaction, as discussed by Bößwetter et al. (2004). Even though these boundary conditions do not forbid the diffusion of the draped magnetic field into the obstacle, this approach allowed to achieve formidable quantitative agreement between simulations and plasma measurements near Mars and Titan (Bößwetter et al., 2004; Simon et al., 2007). We have found that this approach is more sophisticated than setting the fields to zero inside the obstacle, since this introduces a significant energy sink into the system and also violates the $\nabla \cdot \boldsymbol{B}=0$ condition of the magnetic field. As stated by Simon et al. (2006a), the divergence of the magnetic field in our model vanishes.

In all scenarios under consideration, the simulation domain is cubic with an extension of $15 R_{T}$ in each spatial direction. The center of Titan coincides with the center of the simulation domain and denotes the origin $(0,0,0)$ of the coordinate frame. The situation is illustrated in Fig. 1. The time step $\delta t$ of the simulations has been chosen in such way that $1333 \delta t$ correspond to the duration in which the undisturbed magnetospheric plasma would pass through the entire simulation domain (i.e. from $x=-7.5 R_{T}$ to $x=+7.5 R_{T}$ ) one time, i.e. $\delta t=0.24 \mathrm{~s}$. If the magnetospheric upstream conditions did not depend on time, the quasi-stationary state of the simulations would be achieved after a duration that corresponds to about 15 passages of the magnetospheric plasma through the simulation box. Outflow boundary conditions have been applied to all faces of the simulation domain except for the left wall at $x=-7.5 R_{T}$. The left face of the simulation box is treated as an inflow boundary layer, i.e. particles are continously generated according to the constant background values given above. At the grid nodes located in the $\left(x=-7.5 R_{T}\right)$ boundary layer, the electromagnetic fields are given by $\boldsymbol{E}_{0}$ and $\boldsymbol{B}_{0}$.
Several different types of distortions have been applied to the upstream plasma flow. The first setting attempts to mimic highly varying magnetospheric conditions in the vicinity of Titan under which a stationary exospheric tail structure should be unable to develop. In order to realize such a situation, the magnetic field $\boldsymbol{B}_{0}$ in the inflow boundary layer has been set in periodic oscillation according to

$\boldsymbol{B}\left(x=-7.5 R_{T}, t\right)=\boldsymbol{B}_{0} \cos (\omega t)$.

Thus, the orientation of the electric field in the boundary layer is oscillating in the same way:

$\boldsymbol{E}\left(x=-7.5 R_{T}, t\right)=-\boldsymbol{u}_{0} \times \boldsymbol{B}\left(x=-7.5 R_{T}, t\right)$.

During each time step, the directions and values of $\boldsymbol{E}$ and $\boldsymbol{B}$ according to the above equations are "frozen into" the newly generated magnetospheric plasma at the $x=-7.5 R_{T}$ inflow boundary and are then convected towards Titan by the streaming magnetospheric flow. In other words, the distortions are moving towards Titan with the same velocity $\boldsymbol{u}_{0}$ as the magnetospheric plasma does. As can be seen from Eq. (8), although the upstream magnetic field vector oscillates, it remains directed perpendicular to the equatorial plane of Titan. In analogy, the oscillating upstream electric field vector is confined to the satellite's equatorial plane at every instant. The oscillation frequency $\omega$ in Eqs. (8) and (9) has been chosen in such way that a full period $T=2 \pi / \omega$ corresponds to a duration of 1000 time steps. This is comparable to the time that the plasma flow needs for one passage through the simulation box. For comparison, a 3.5 times smaller oscillation frequency has also been tested. A further reduction of the oscillation frequency would make it impossible to achieve a quasi-stationary ${ }^{1}$ state in a reasonable computing time.

If the distorted electromagnetic fields were chosen in a different way, i.e. if three non-vanishing components were introduced (e.g. by a rotation), the ionospheric tail would exhibit an extremely complex three-dimensional structure, which makes it impossible to gain any access to the involved physical processes. The distortions introduced above allow a straightforward analysis of how Titan's tail structure in the asymmetry plane (defined by $\boldsymbol{u}_{0}$ and $\boldsymbol{E}_{0}$ ) is altered if the upstream conditions forbid the development of a stationary pick-up tail. Note that, despite the oscillation of $\boldsymbol{E}$ and $\boldsymbol{B}$, the pick-up velocity

$\boldsymbol{u}_{p}=\boldsymbol{E} \times \boldsymbol{B} / B^{2}$

still points downstream in $(+x)$ direction. The model presented above does not include an oscillation of the magnetospheric plasma densities. Although a relatively high number of about 40 magnetospheric macroparticles is initially placed in each cell at the inflow boundary layer, oscillations

\footnotetext{
${ }^{1}$ As will be discussed below, within the framework of this paper, the term "quasi-periodic" state is more appropriate.
} 
Table 1. Overview of the discussed simulation scenarios. The time step $\delta t$ of the simulations has been chosen in such way that $1333 \delta t$ correspond to one passage of the plasma through the entire simulation domain from $x=-7.5 R_{T}$ to $x=+7.5 R_{T}$, i.e. $\delta t=0.24 \mathrm{~s}$. As discussed in the text, this is significantly smaller than the gyroperiods of the involved pick-up species.

\begin{tabular}{ll}
\hline Simulation run & Magnetic field distortion \\
\hline$\# 1$ & none, stationary case \\
$\# 2$ & $\boldsymbol{B}\left(x=-7.5 R_{T}, t\right)=\boldsymbol{B}_{0} \cos (\omega t)$, \\
& $T=2 \pi / \omega=1000 \delta t$ \\
$\# 3$ & $\boldsymbol{B}\left(x=-7.5 R_{T}, t\right)=\boldsymbol{B}_{0} \cos (\omega t)$, \\
& $T=2 \pi / \omega=3500 \delta t$ \\
$\# 4$ & $\boldsymbol{B}\left(x=-7.5 R_{T}, t\right)=\boldsymbol{B}_{0}(1+\cos (\omega t))$, \\
& $T=2 \pi / \omega=3500 \delta t$ \\
$\# 5$ & tangential discontinuity \\
\hline
\end{tabular}

of the density have shown to be impossible to handle from the standpoint of numerical stability.

As stated in our companion paper, the thermal velocity of the particles inside Saturn's magnetosphere exceeds the upstream flow speed $u_{0}$ by more than a factor of 2 . Due to this high temperature, test runs have shown that it is impossible to self-consistently incorporate a "real" plasma wave that propagates towards Titan. The wave is damped almost instantaneously, i.e. after having passed a distance of less than $0.5 R_{T}$. The reason for this is not only the physical damping in the hot magnetospheric plasma, but also the "numerical" damping due to the finite number of particles. Therefore, the disturbed upstream conditions presented above represent the best compromise that can (currently) be achieved with a semi-kinetic model.

Test runs have also shown that this is not a specific problem of the Titan scenario, but a simulation study of nonstationary pick-up conditions in the solar wind, e.g. at Mars or comets, would face the same restrictions. Since in this case, the interaction gives rise to a bow shock in front of the obstacle, it is even nearly impossible for the simulated distortions to reach the region downstream of the bow shock, where e.g. the asymmetric Martian pick-up tail is located under stationary conditions (Bößwetter et al., 2004). Since Titan's plasma interaction does not lead to the formation of a shock, it is significantly "easier" for the distortions to reach and interact with the pick-up tail. The Titan scenario therefore provides a unique plasma laboratory in which the resulting physical phenomena can be examined.

Of course, despite allowing to gain straightforward access to the physics of ion pick-up under varying upstream conditions, the field (8) is artificial in such way that a complete reversal of $\boldsymbol{B}_{0}$ cannot occur inside a planetary magnetosphere. Apparently, an ansatz for the upstream field in the left-hand boundary according to

$\boldsymbol{B}\left(x=-7.5 R_{T}, t\right)=\boldsymbol{B}_{0}+\boldsymbol{B}_{1} \cos (\omega t)$

would be more sophisticated. Even though such a treatment may be closer to reality, identifying the involved physical processes is more difficult in that situation. However, the discussion shall also focus on such a scenario, using a boundary field according to

$\boldsymbol{B}\left(x=-7.5 R_{T}, t\right)=\boldsymbol{B}_{0}(1+\cos (\omega t))$,

where $\omega=2 \pi /(1000 \delta t)$ or $\omega=2 \pi /(3500 \delta t)$, respectively. In this case, the constant background field $\boldsymbol{B}_{0}$ is modulated with the distortion of amplitude $\boldsymbol{B}_{0}$, i.e. the field magnitude oscillates between 0 and $2 B_{0}$.

In the second part of our simulation study, the influence of tangential discontinuities on Titan's tail structure has been investigated. At first, the simulation was allowed to achieve a quasi-stationary state in which the ion tail is fully developed. Then, the magnetic field direction in the inflow boundary layer $\left(x=-7.5 R_{T}\right)$ has been suddenly rotated by an angle $\phi$ and has again been kept constant in the new direction until stationarity was re-achieved. The modified field orientation is convected towards Titan by the newly generated plasma flow. The characteristic time scales on which the ionospheric tail structure adapts to the re-oriented pick-up fields have been investigated. Two different values of $\phi$ have been tested: At first, the magnetic field has been rotated by 180 degrees, i.e.

$\boldsymbol{B}_{0} \rightarrow-\boldsymbol{B}_{0}$.

Hence, the electric field $\boldsymbol{E}_{0}$ also reverses its direction, but it remains parallel to Titan's equatorial plane. This "extreme" situation is meant to provide a general impression of the processes that occur when Titan crosses Saturn's magnetopause. In the second scenario, a rotation angle of $\phi=90^{\circ}$ has been applied. After the rotation, the undisturbed magnetic field points in $(-y)$ direction. Consequently, $\boldsymbol{E}_{0}$ becomes parallel to the $(x, z)$ plane. Thus, the planes defined by $\left(\boldsymbol{u}_{0}, \boldsymbol{B}_{0}\right)$ and $\left(\boldsymbol{u}_{0}, \boldsymbol{E}_{0}\right)$ have simply been "exchanged". As discussed by Simon et al. (2006b, 2007b), in the $\left(\boldsymbol{u}_{0}, \boldsymbol{B}_{0}\right)$ plane, the Titan tail should possess a symmetric structure, whereas the tail structure in the $\left(\boldsymbol{u}_{0}, \boldsymbol{E}_{0}\right)$ plane features a strong asymmetry. An overview of the analyzed model cases is given in Table 1.

Finally, a brief remark on the characteristic time scales occurring in the simulations seems appropriate. The gyroperiods

$T_{g}=\frac{m}{e B}$

of the three pick-up species included in the model are $T_{g}\left(\mathrm{~N}_{2}^{+}\right)=58.46 \mathrm{~s}, T_{g}\left(\mathrm{CH}_{4}^{+}\right)=33.41 \mathrm{~s}$ and $T_{g}\left(\mathrm{H}_{2}^{+}\right)=2.08 \mathrm{~s}$. These scales are significantly larger than the time step $\delta t=0.24 \mathrm{~s}$, i.e. the gyration of all three pick-up species is well resolved by the model. The periods of the field distortions are given by $1000 \delta t=241 \mathrm{~s}$ and $3500 \delta t=845 \mathrm{~s}$. In other 

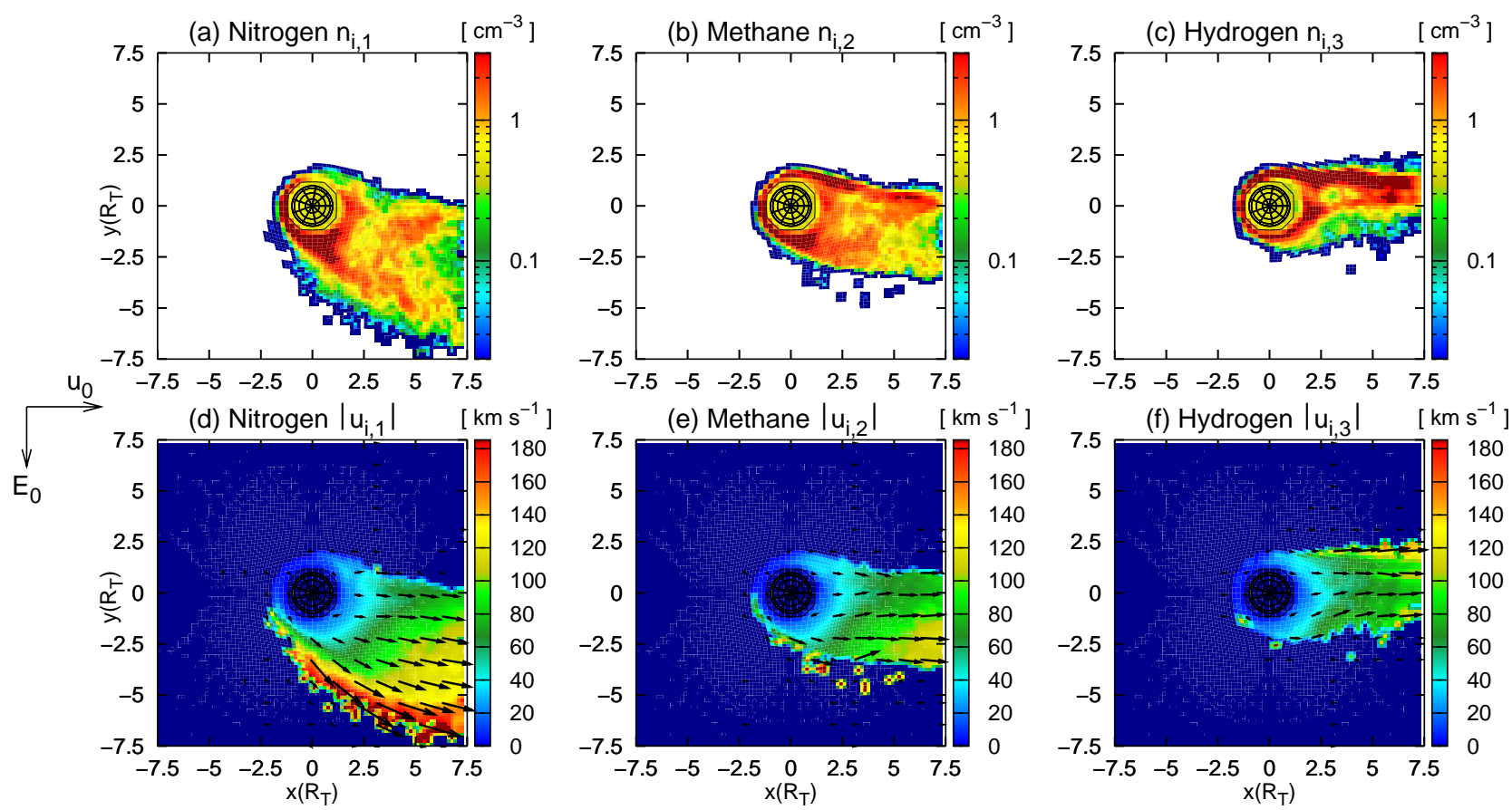

Fig. 2. Run \#1: Titan's plasma environment at 18:00 Saturnian local time. The figure shows the ionospheric plasma parameters for a cut through Titan's equatorial plane. Specifically, the figure displays the densities (a) of molecular nitrogen ions (subscript $i, 1)$, (b) of methane ions (subscript $i, 2)$ and (c) of molecular hydrogen ions (subscript $i, 3$ ). The corresponding plasma velocities are shown in panels (d) to (f). Near the outer flank of the tail in the $(y<0)$ hemisphere, where the ions are strongly accelerated, their densities can practically be neglected. The figure has been obtained from Simon et al. (2007b).

words, one quarter of a wave period (which is the time scale of field variation for the pick-up ions) is at least comparable to the gyroperiod of the heaviest species, while it clearly exceeds those of the two lighter ones. Another time scale occurring in the simulations is the magnetic diffusion time. Since no boundary conditions are imposed on the electromagnetic field, $\boldsymbol{B}$ can be transported into the ionosphere and even inside the planet by means of numerical diffusion. However, as we have demonstrated in our companion studies (cf. especially Simon et al., 2006b; Simon et al., 2007, and Bößwetter et al., 2004; Bößwetter et al., 2007), this effect takes practically no influence on the outcome of the simulation, i.e. the characteristic signatures observed by spacecraft flybys are reproduced quite well. Especially in the case of Mars, the position of the shock and the ramside magnetic draping boundary have shown to be quantitatively reproducible, despite the diffusion effect. The magnetic diffusion scale clearly exceeds the characteristic time scales of the included field distortions.

\section{Titan's tail structure: the stationary state}

The following analysis will mainly concentrate on the structure of Titan's ionospheric tail in the satellite's equatorial plane which is identical to the $(x, y)$ plane of the coordi- nate system. This plane is defined by the fields $\boldsymbol{u}_{0}$ and $\boldsymbol{E}_{0}$. Figure 2 shows the ionospheric tail structure in this plane for the case of stationary upstream conditions. Some key aspects shall be briefly pointed out:

- Asymmetric tail structure: The tail is confined to the hemisphere in which $\boldsymbol{E}_{0}$ points away from Titan.

- Mass spectrometer effect: The newly generated ionospheric ions are moving on cycloidal trajectories. The extension of the cycloidal arcs in $(y<0)$ direction depends linearly on the mass of the respective ion species. Therefore, the diameter of the molecular nitrogen tail (species $i, 1)$ clearly exceeds the diameter of the methane tail (species $i, 2$ ), whereas the light molecular hydrogen ions (species $i, 3$ ) are confined to a narrow region directly behind Titan.

- Suppression of pick-up of light species: The velocity achieved at the $(y<0)$ flank of the $\mathrm{N}_{2}^{+}$tail clearly exceeds the speed achieved by methane and hydrogen. Directly behind Titan, the nitrogen velocity is significantly smaller than at the tail's $(y<0)$ flank. The accumulation of slow nitrogen ions directly behind Titan yields a reduction of the convective electric field strength in the 

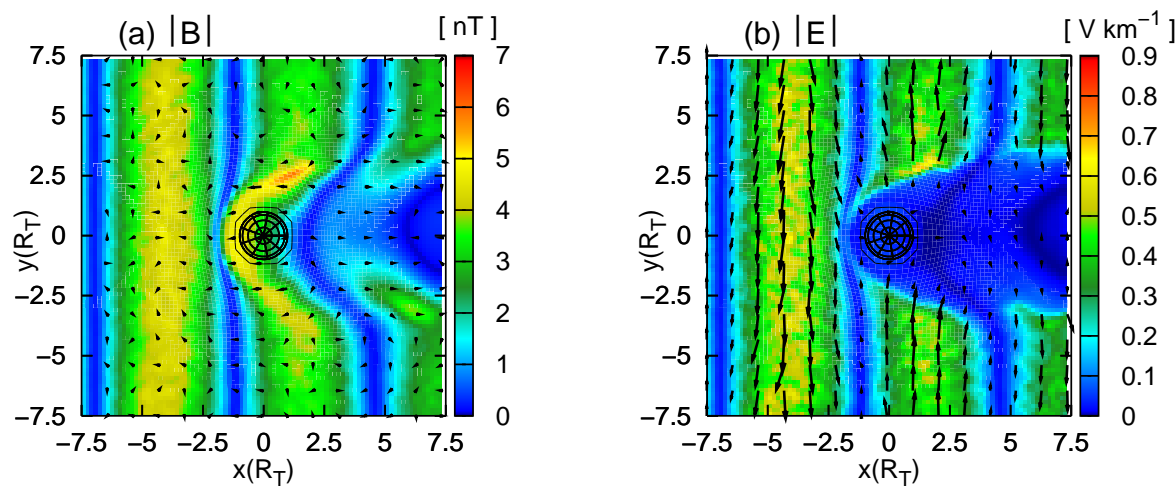

Fig. 3. Run \#2: Titan's plasma environment when the satellite is exposed to a periodic distortion according to Eqs. (8) and (9). The period $T=2 \pi / \omega$ of the distortion is $T=1000 \delta t$, where $1333 \delta t$ correspond to the duration in which the magnetospheric plasma would pass through the simulation box one time. The plot shows a snapshot of the electromagnetic fields in Titan's orbital plane.
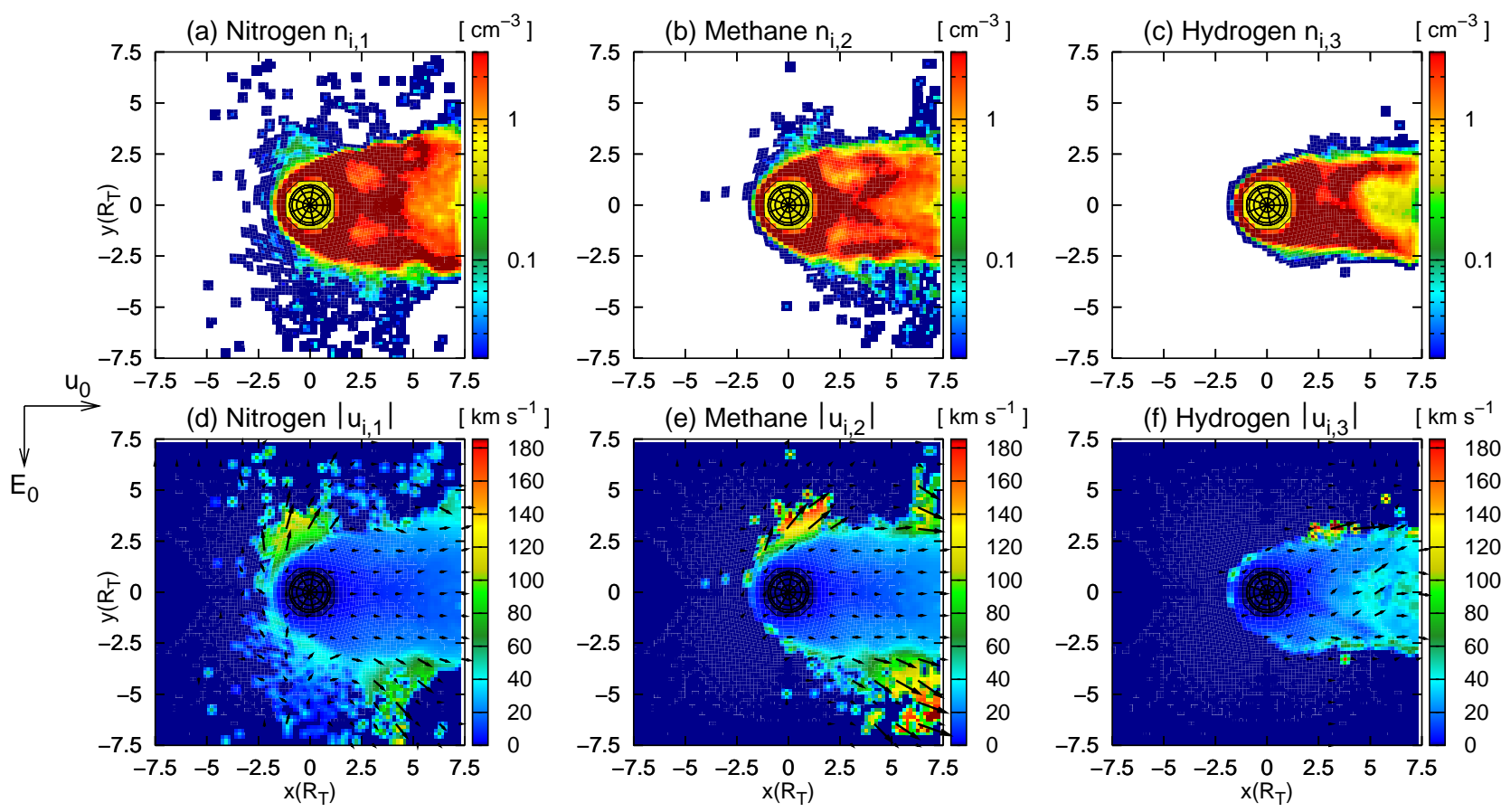

(e) Methane $\left|\mathrm{u}_{\mathrm{i} 2}\right| \quad\left[\mathrm{km} \mathrm{s}^{-1}\right]$
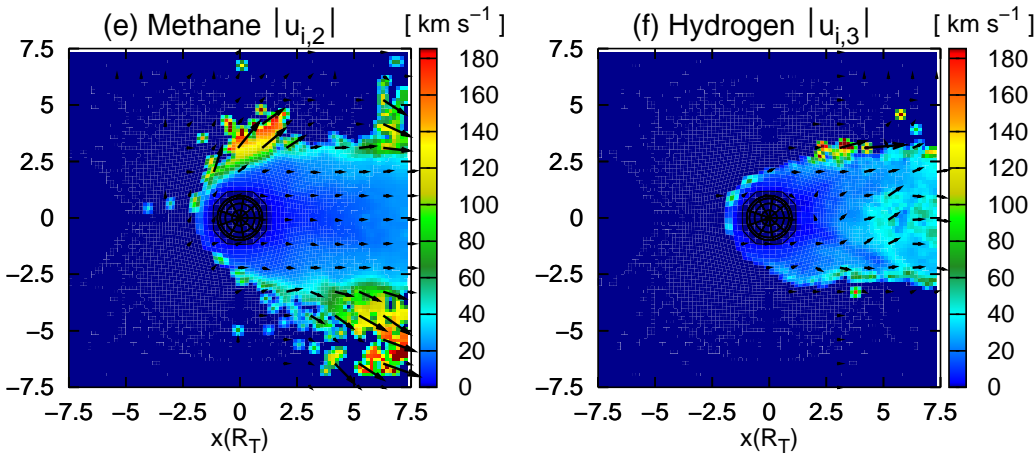

Fig. 4. Run \#2: Titan's plasma environment when the satellite is exposed to a periodic distortion according to Eqs. (8) and (9). The period $T=2 \pi / \omega$ of the distortion is $T=1000 \delta t$, where $1333 \delta t$ correspond to the duration in which the magnetospheric plasma would pass through the simulation box one time. The plot shows the ionospheric plasma densities $n$ and velocities $u$ that correspond to the preceding figure. The subscript $i, 1$ denotes the parameters of molecular nitrogen, whereas $i, 2$ and $i, 3$ represent the methane and molecular hydrogen component, respectively.

central wake. Due to their smaller masses and therefore smaller gyroradii, the tails developed by $\mathrm{CH}_{4}^{+}$and $\mathrm{H}_{2}^{+}$are almost completely located inside this nitrogeninduced electric field cavity. Therefore, the transport of the two lighter species away from Titan is less efficient than the pick-up of the nitrogen particles.
None of the three effects described above occurs in Titan's polar plane, which is defined by $\boldsymbol{u}_{0}$ and $\boldsymbol{B}_{0}$. In this plane, the pick-up tail exhibits a narrow and symmetric structure. At either side, it is confined by one of Titan's magnetic lobes. 


\section{Influence of periodic field variations: short oscillation period}

We consider an electromagnetic field distortion according to Eqs. (8) and (9). At first, we shall discuss the simulation with $T=2 \pi / \omega=1000 \delta t$. In this situation, the characteristic time scale of the field perturbation differs from the traveling time of the plasma through the box by only a factor of 1.3 .

Of course, this kind of simulation will not achieve a "real" quasi-stationary state in which the plasma quantities are constant in time. However, after a few passages of the plasma through the simulation domain, the structure of Titan's ionospheric tail exhibits the same periodicity as the impinging distortion itself. A snapshot of the simulation results in this "quasi-periodic" state is shown in Figs. 3 and 4. For a cut through the equatorial plane, Fig. 3 illustrates the electromagnetic fields, whereas the structure of Titan's ionospheric tail can be seen in Fig. 4. Each of the three ionospheric tails is characterized by a broad, nearly symmetric region where the ionospheric plasma density clearly exceeds the density of the undisturbed magnetospheric plasma. The outer boundary of this structure is parabolically shaped and denotes a sharp density jump by more than one order of magnitude. Inside this central tail region, the ionospheric plasma velocity almost vanishes and achieves values of less than $30 \mathrm{~km} / \mathrm{s}$, as can be seen in Fig. $4 \mathrm{~d}$ to $\mathrm{f}$. Along the outer flanks of the nitrogen and methane tails, diffuse regions of increased ionospheric plasma velocity are attached. As displayed in Fig. $4 \mathrm{c}$ and $\mathrm{f}$, such signatures do not manifest in the flow pattern of the molecular hydrogen ions, i.e. no hydrogen ions at all can be found outside the parabolically shaped boundary layer. While the diffuse structures attached to the nitrogen and methane tails exhibit strong time variations, the flow pattern in the parabolically shaped region has shown to remain nearly unaffected by the strongly varying upstream conditions. In the following, the inner region of the tail which is surrounded by the parabolic boundary layer will be called the "central tail".

As stated above, the plasma inside the central tail region is not much affected by the distortions in the adjacent magnetospheric flow. In fact, this region has shown to be able to shield itself against any kind of short-time variation in the ambient magnetospheric plasma by forbidding the magnetospheric pick-up fields to penetrate into its interior. As can be seen from Fig. 3b, the central tail region does not only manifest in the ionospheric ion densities, but it also leaves a strong imprint on the electric field in Titan's equatorial plane. Inside the central tail region, the electric field strength nearly vanishes. These structures arise from some kind of feedback mechanism which can be understood as follows: in the immediate vicinity of Titan, huge amounts of newly generated, slow ionospheric particles are accumulated. Due to their massive concentration, these slow particles cause the convective electric field near Titan

$\boldsymbol{E}_{c}=-\left\{\sum_{j} \frac{n_{j}}{n_{\text {total }}} \boldsymbol{u}_{j}\right\} \times \boldsymbol{B}$

to vanish almost completely. In this expression, $n_{j}$ and $\boldsymbol{u}_{j}$ denote the densities and velocities of the different ion species, while $n_{\text {total }}=\sum_{j} n_{j}$ is the total ion density at a certain position. In other words, the efficiency of the pickup process near Titan is drastically reduced. When, subsequently, newly generated ions enter the wake, they are practically not accelerated, since they experience the reduced convective field strength in the cavity. This makes them accumulate near Titan to an even higher degree, i.e. the electric field in the wake and therefore, the efficiency of the pick-up process is reduced even further. The slow movement of these particles in the downstream direction leads to the formation of the central tail region.

As also illustrated by Eq. (15), the electric field term $\boldsymbol{E}_{c}$ is made up of contributions of all ion species included into the model. Since in the central tail region, the ionospheric particles are predominant ones, the distorted magnetospheric electromagnetic fields are unable to penetrate into this region. Of course, the magnetospheric "background plasma" gives a minor contribution to the electric and magnetic field, but both quantities are drastically reduced with respect to the upstream values. Thus, the central tail shields itself against the distortions by means of the high concentration of slow ionospheric particles. Only at the flanks of the central tail, where the ionospheric density diminishes and becomes comparable to the magnetospheric upstream density, the distorted electromagnetic fields are able to remove some of the accumulated ionospheric particles. The distortions cannot penetrate through the entire tail instantaneously, but they must gain access to the central tail region from outward to inward by removing particles from its flanks. This can be seen in Fig. $4 a, b, d$ and e. If, in a certain region, the magnetospheric electric field does not change its direction for some time, a cycloidal pick-up tail like the one shown in Fig. 2 starts to be formed. However, since the field direction does not remain constant long enough for a full cycloid to be developed, the formation of this tail is interrupted. Therefore, the formation of the diffuse "plasma clouds" in the nitrogen and methane flow patters can be ascribed to the removal of plasma from the outer boundaries of the central tail due to incomplete pick-up.

The weak influence of the magnetospheric fields on the inner tail region has been observed in our foregoing studies using stationary upstream conditions as well. In that situation, the reduction of the electric field strength directly behind the obstacle due to the massive presence of slow methane and nitrogen ions leads to a suppression of the hydrogen pick-up process. A similar dependency of the individual tail structures on the particle mass manifests in Fig. 4 as well. The erosion of the central tail by the distorted magnetospheric 

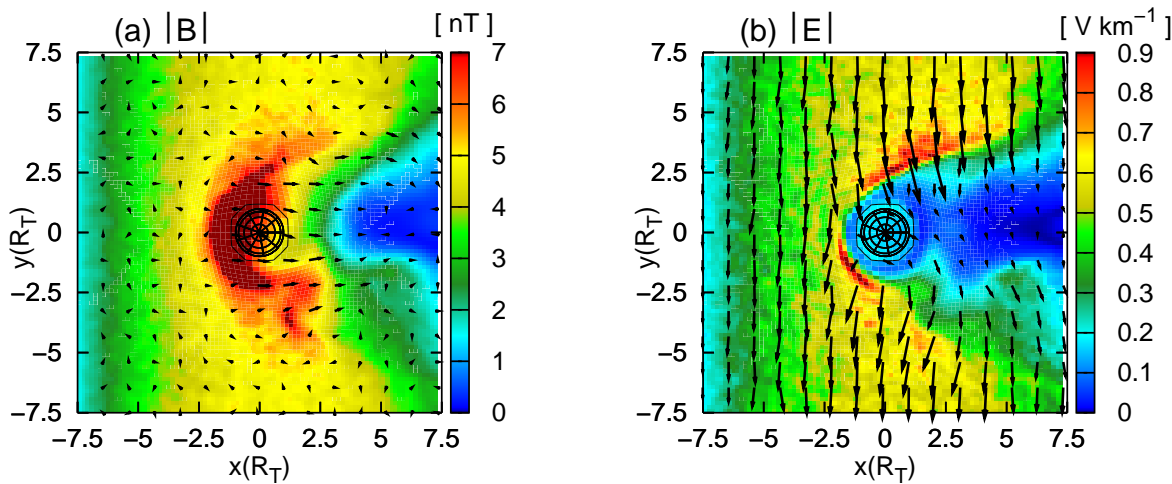

Fig. 5. Run \#3: Titan's plasma environment when the moon is exposed to a periodic distortion according to Eqs. (8) and (9). The period $T=2 \pi / \omega$ of the distortion is $T=3500 \delta t$, where $1333 \delta t$ correspond to the duration in which the magnetospheric plasma would pass through the simulation box one time. The plot shows a snapshot of the electromagnetic fields in Titan's orbital plane.
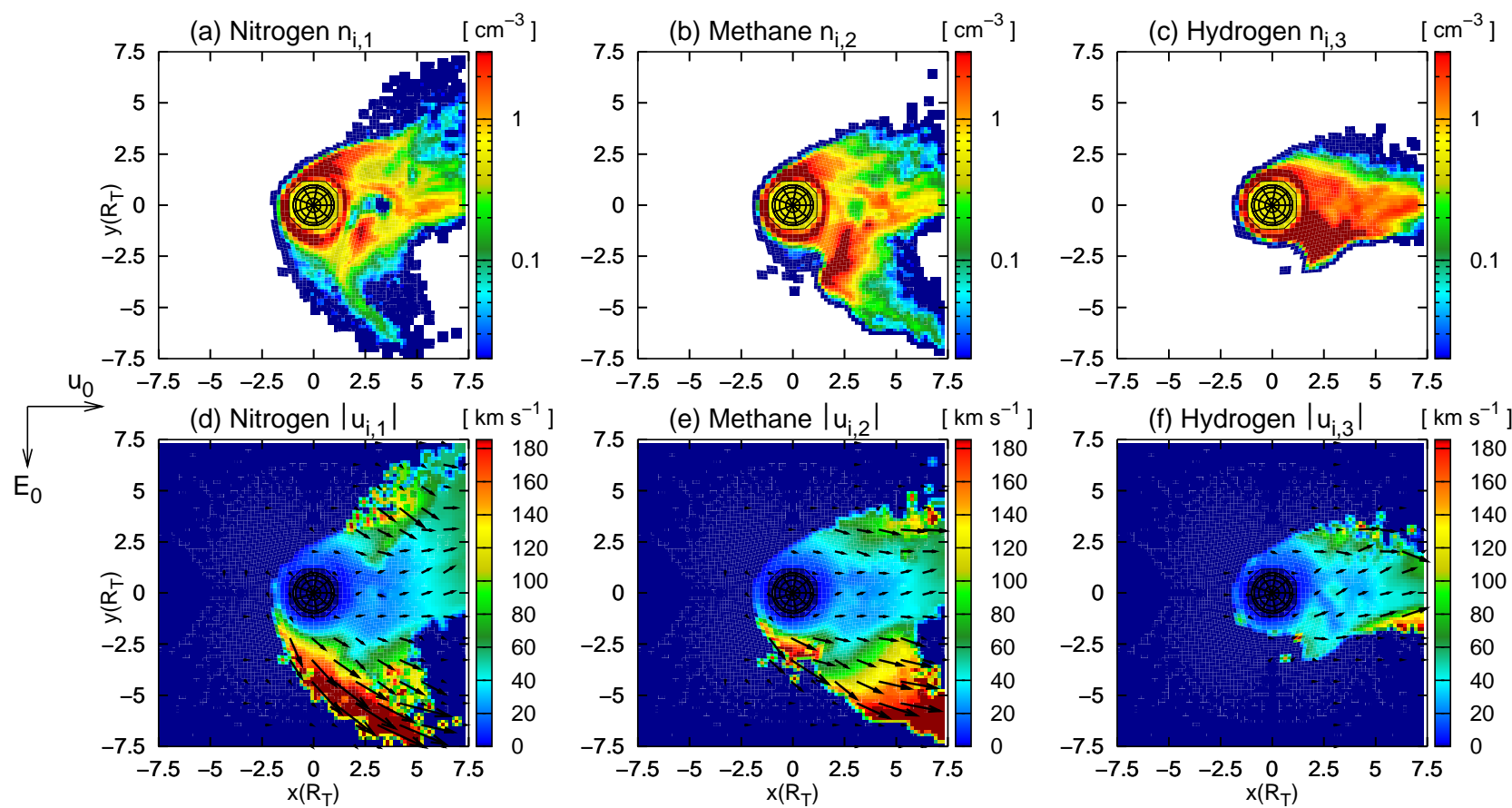

Fig. 6. Run \#3: Titan's plasma environment under the influence of a periodic distortion according to Eqs. (8) and (9). The period $T=2 \pi / \omega$ of the distortion is $T=3500 \delta t$, where $1333 \delta t$ correspond to the duration in which the magnetospheric plasma would pass through the simulation box one time. The plot shows the ionospheric plasma densities $n$ and velocities $u$ that correspond to the foregoing figure.

fields manifests only in the nitrogen and the methane tail, but the central tail formed by the light hydrogen ions is apparently not influenced by this effect. Although the varying upstream conditions prevent the formation of the magnetospheric mass spectrometer, the lightest species, possessing the smallest gyroradius, still seems to be "protected" from the pick-up process by the two heavier ones. This may be considered a remnant of the mass spectrometer formed in the stationary state. In any case, the simulation confirms the hypothesis of Luhmann (1996) that the spatially dispersive tail structure can only be formed under stationary upstream conditions.

Before proceeding with the analysis, it should be noted that the shielding of an obstacle's ionospheric tail against the ambient electromagnetic fields is also typical of the plasma environment of a weak comet. Bagdonat (2005) as well as 
Motschmann and Kührt (2006) have shown that even in the regime of a weakly outgassing nucleus, a comet's heavy ion tail is surrounded by a sharply pronounced boundary layer, inside which the solar wind fields nearly vanish. The formation of this structure is explained in terms of the same feedback mechanism that gives rise to the central ion tail in the Titan scenario.

\section{Influence of periodic field variations: long oscillation period}

In the following, we shall focus on the second scenario, assuming the period of the oscillation to be about a factor of 3.5 larger than above. The oscillations in the left-hand boundary of the box are again given by Eqs. (8) and (9), respectively. In this case, the relatively large intervals of spatially constant electromagnetic field direction make it "easier" for the ionospheric tail orientation to adapt to the variable upstream conditions.

A snapshot of the simulation results is shown in Figs. 5 and 6. Again, we show the situation after the modification of the tail structure has achieved a quasi-periodic state. As can be seen from Fig. 5, the snapshot refers to a situation where, due to the large period of the oscillation, the electric field direction in the entire simulation domain is the same. The field is oriented antiparallel to the y-axis. At the left-hand boundary of the simulation domain, the next half period of the wave in which the directions of the pick-up fields are reversed, is about to enter the box. The ionospheric tail structure is shown in Fig. 6. Titan's plasma wake exhibits some kind of split signature with one fragment of the tail being located in the $(y>0)$ hemisphere and the other one being shifted in the $(y<0)$ direction. Only in the $(y<0)$ part of the tail, the ions are strongly accelerated due to the pick-up process. The nitrogen and methane tail structures that start to develop in this hemisphere are similar to the cycloidal tails displayed in Fig. 2a and b. As can be seen from Fig. $6 \mathrm{~d}$, in the $(y<0)$ part of the tail, the ions are accelerated in the momentary direction of the electric field, i.e. away from Saturn. Although not being as prominent as in the preceding simulation scenario, the central tail region of reduced ionospheric plasma velocity and increased density is still identifiable for all three species. However, the sharp outer boundary of this structure has vanished completely. The density decreases steadily from the central tail to the two tail fragments that are attached to this region, the transition between both regions being rather diffuse.

Due to the large time scale of the fluctuations, the ambient magnetospheric electromagnetic fields are able to erode the central tail region more efficiently than in the case of a fast fluctuating disturbance with a period of only $1000 \delta t$. In the case of a small wave period, ions are detached from the central tail near its flanks. However, the period of the wave and therefore, the constant orientation of the pick-up fields in a certain region, is too short to transport these ions sufficiently far away from Titan. In the following half period, the detached ions are accelerated back into the direction of the central tail by the reversed fields and are re-incorporated into it, while the opposite side of the tail is now exposed to erosion. In other words, when the period of the distortion is sufficiently small, any kind of erosion of the central tail is compensated almost instantaneously by the re-incorporation of the picked-up ions into the tail. The larger is the wave period, the longer are the magnetospheric fields at the central tail's flank oriented in the same direction and the deeper can they penetrate into the central tail region. For this reason, the boundary of the central tail in Fig. 6d does no longer denote a sharp density decrease. In the $(y>0)$ hemisphere, large regions are covered by heavy ions that have been dragged there during the preceding half wave period in which $\boldsymbol{E}$ was pointing towards Saturn. Of course, in the situation shown in Fig. 6, this heavy ion cloud is accelerated back to the central tail, but the time scale for the re-incorporation is significantly larger than in the case of a small wave period. The formation of the cycloidal pick-up tail during a certain halfperiod of the wave is more complete than in the $T=1000 \delta t$ scenario. Therefore, in the subsequent half-period, it takes the reversed fields longer to re-collect the detached ions and to drag them back to Titan, while the formation of the pick-up tail commences at the other side of the satellite. The nearly instantaneous re-incorporation and compensation of tail erosion must likely be ascribed to the high spatial symmetry of the periodic oscillations.

Another snapshot of the situation in Titan's equatorial plane at a later point in time is shown in Figs. 7 and 8. The half wave of reversed $\boldsymbol{E}$ and $\boldsymbol{B}$ which was about to enter the box in the preceding snapshot now covers the entire tail region of Titan. These figures illustrate quite well how the tail in the $(y<0)$ hemisphere is retracted when the direction of the pick-up electric field is reversed. Again, the figure shows a situation in which the electric field in the entire equatorial plane points in the same direction, i.e. towards Saturn. However, the removal of the tail fragment in the "wrong" hemisphere (which, in this case, is the $(y<0)$ hemisphere) has not yet proceeded as far as in the foregoing snapshot. The electric field forces the ionospheric tail to shift into the $(y>0)$ hemisphere. As can clearly be seen from Fig. 8d, the $(y>0)$ flank of the newly developing nitrogen tail exhibits a cycloidal shape. In the $(y<0)$ hemisphere, the original cycloidal pick-up tail is dismantled by the reversed electric field. As shown in Fig. 8d, the flow pattern in the $(y<0)$ hemisphere is characterized by two different processes: Although behind the $\left(x>4 R_{T}\right)$ line, the ionospheric $\mathrm{N}_{2}^{+}$ions are already exposed to the reversed electric field, the field magnitude is by far not as strong as in the peak of the distortion which is currently located in the $\left(x<4 R_{T}\right)$ half space. Therefore, in the $\left(x>4 R_{T}\right)$ region, the ionospheric particles in the anti-Saturn-facing hemisphere are decelerated, but nonetheless, they are still able to maintain their original flow 

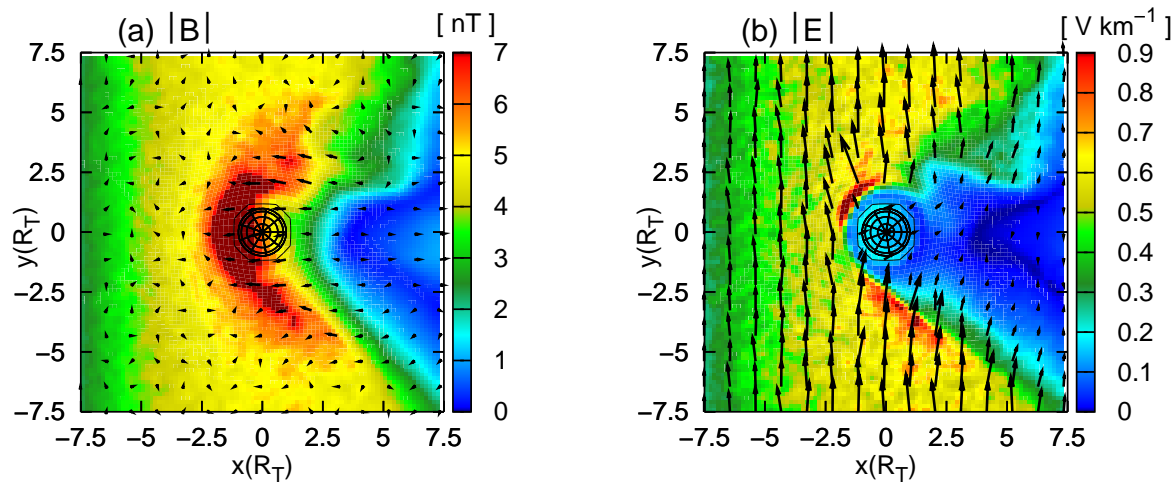

Fig. 7. Run \#3: Titan's plasma environment when the moon is exposed to a periodic distortion according to Eqs. (8) and (9). The period $T=2 \pi / \omega$ of the distortion is $T=3500 \delta t$, where $1333 \delta t$ correspond to the duration in which the magnetospheric plasma would pass through the simulation box one time. The plot shows another snapshot of the electromagnetic fields in Titan's orbital plane. Compared to the preceding snapshot, the field direction is reversed. In the state displayed here, the region of increased field strength is located a little closer to the left-hand boundary of the simulation domain than in the preceding snapshot (compared to Fig. 5, the light blue region near the left-hand boundary of the box is missing here).

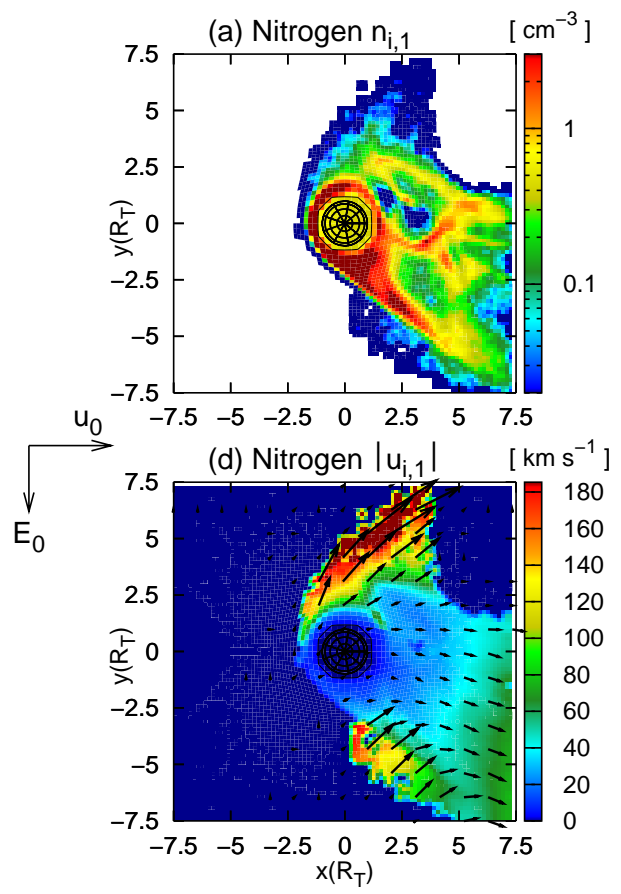

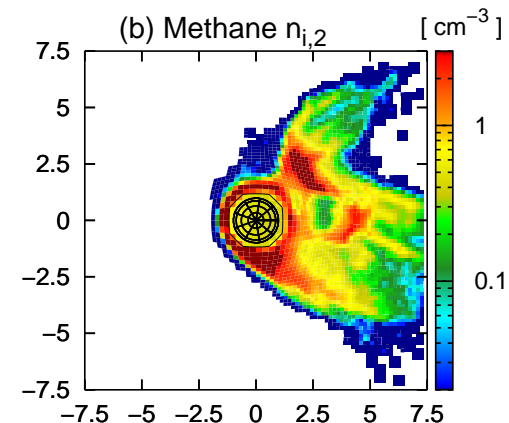

(e) Methane $\left|\mathrm{u}_{\mathrm{i}, 2}\right| \quad\left[\mathrm{km} \mathrm{s}^{-1}\right]$

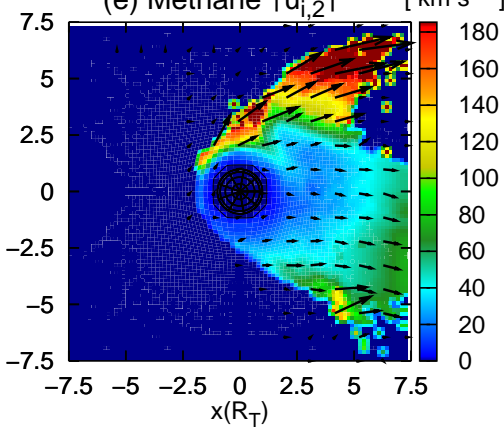

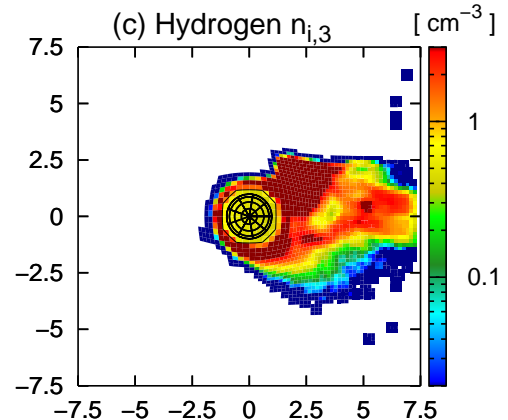

(f) Hydrogen $\left|\mathrm{u}_{\mathrm{i}, 3}\right| \quad\left[\mathrm{km} \mathrm{s}^{-1}\right]$

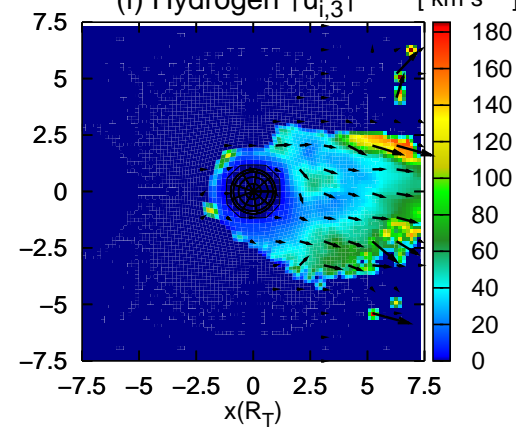

Fig. 8. Run \#3: Titan's plasma environment under the influence of a periodic distortion according to Eqs. (8) and (9). The period $T=2 \pi / \omega$ of the distortion is $T=3500 \delta t$, where $1333 \delta t$ correspond to the duration in which the magnetospheric plasma would pass through the simulation box one time. The plot shows the ionospheric plasma densities $n$ and velocities $u$ that correspond to the foregoing figure.

direction. At the forward flank of the $(y<0)$ tail, on the other hand, the nitrogen ions already experience the full strength of the field reversal, leading to a strong acceleration of the detached ion population in $(y>0)$ direction (cf. Fig. 8d). The effect of the field reversal is clearly visible in the $(y<0)$ ni- trogen flow pattern. This also illustrates that when the distortion arrives at Titan, it is not instantaneously able to affect the ionospheric particles in the entire tail region, but it must instead erode the tail from outward to inward. Initially, the $\mathrm{N}_{2}^{+}$tail in the $(y<0)$ hemisphere possessed a cycloidal 
shape, like the flank of the newly generated ion population in the $(y>0)$ hemisphere. As can be seen from Fig. 8 as well as in the imprint that the ionospheric ions leave in the electric field topology (cf. Fig. 7b), the $\mathrm{N}_{2}^{+}$tail flank now possesses a nearly straight shape, while the inner region of reduced $\mathrm{N}_{2}^{+}$ velocity is still clearly identifiable. The distortion starts to erode the $\mathrm{N}_{2}^{+}$tail at its outer flank, but the characteristic time that it needs to penetrate into the inner tail region is significantly larger than the traveling time scale of the perturbed field itself.

As displayed in Fig. 8b, the cycloidal shape of the methane tail's flank in the $(y<0)$ half space is still quite well identifiable. Besides, despite the lower mass of methane, the effect of the reversed fields near the $(y<0)$ flank of the methane tail is by far not as pronounced as the acceleration of the nitrogen ions. In contrast to the behaviour of the nitrogen component, there are so far no methane clouds in the $(y<0)$ hemisphere that are strongly accelerated in positive y-direction. As stated above, in the case of stationary upstream conditions, the methane tail is completely located inside the electric field cavity generated by the massive accumulation of slow nitrogen ions. The reason for this is the larger gyroradius of nitrogen. Therefore, the reversed fields have to dismantle the $(y<0)$ flank of the heavy $\mathrm{N}_{2}^{+}$tail at first, before they can "start" to erode the methane tail. In other words, the tail developed by the heaviest ionospheric species is able to exert some kind of shielding effect on the lighter ones. As can be seen from the flow pattern of the light ionospheric $\mathrm{H}_{2}^{+}$ ions in Fig. 8f, the velocity vectors of this component in the $(y<0)$ half space have not yet started to reverse their direction. The tails developed by the three species in the $(y<0)$ hemisphere during the foregoing half period are not retracted simultaneously, but in the same sequence as they occur in the ionospheric mass spectrometer. At first, the heavy nitrogen tail is deformed by the distortion, i.e. at its outer flank, particles are accelerated in $(y>0)$ direction. This goes along with a deformation of the electric field cavity formed by $\mathrm{N}_{2}^{+}$. As shown in Fig. 2 for the case of stationary upstream conditions, in the major pick-up region of the $(y<0)$ hemisphere, the ionospheric densities are relatively small, and so is their contribution to the electric field. Therefore, it is relatively "easy" for the field distortions to start the tail erosion in this region. After some time, the protection of the methane tail by the more extended nitrogen tail has been removed. Then, the reversed electric field starts to erode the methane tail, and finally, the particles in the $\mathrm{H}_{2}^{+}$tail are affected.

The simulation results discussed above show that the larger is the oscillation period, the smaller is the region in which the heavy ion tail is completely shielded from the ambient field distortions. If the period of the oscillation was increased even further, the central tail (characterized by increased ionospheric particle density, reduced electric field strength and reduced flow speed) would vanish completely. In this situation, a broad heavy ion cloud that covers both hemispheres would be formed in the wake region, the dynamics of the ionospheric particles inside the entire wake being highly susceptible to the changes in the upstream conditions. In other words, a short-period oscillation in the upstream electromagnetic fields causes less significant variations in the global tail structure than a long-period disturbance.

\section{Influence of periodic field variations: modulated background field}

In the preceding section, the magnetospheric upstream conditions were assumed to be highly perturbed, thus preventing the formation of a stationary pick-up tail. The necessary magnetospheric distortions have been modeled by permanently changing the direction of the upstream fields, assigning the situation a somewhat artificial character. Therefore, this section shall focus on the case of a distortion that is superimposed on a steady upstream field according to Eq. (12). The magnitude of the periodic perturbation, however, is identical to the upstream field value, i.e. the field varies between $2 B_{0}$ and 0 . Hence, even though the amplitude of the magnetic field undergoes permanent changes, its direction remains the same at every point of the simulation domain. In the following, we shall mainly focus on the simulation run with the long wave period $(T=3500 \delta t)$. Since the areas of reduced or enhanced field strength are larger than in the $T=1000 \delta t$ run, the involved physical processes can be easier identified.

Some snapshots of the situation are shown in Figs. 9 and 10. Figure 10 shows the densities and velocities in the tail when the near-Titan region is almost completely covered by a minimum of the distortion. The corresponding electromagnetic field topology can be seen in Fig. 9. For the case of Titan's tail being completely located in a region of increased field strength, the ionospheric plasma parameters are not shown since the involved physical effects are basically the same as already displayed. As can be seen from the density plots, the overall structure of the ionospheric tail is only slightly affected, if the upstream fields change their magnitude, but not their direction. The ionospheric plasma population is completely confined to the $(y<0)$ half space. Only the extension of the tails in $(y<0)$ direction is changed according to the period of the distortions. In general, a reduction of the ambient magnetic field strength goes along with an increase of the local ion gyroradius, i.e. the extension of the cycloidal pick-up trajectories in $(y<0)$ direction is enlarged. On the other hand, when the tail is exposed to a region of increased field strength, the local ion gyroradii are smaller than in the undisturbed case (cf. Fig. 2).

However, the tail developed by the heaviest species is again able to protect the two lighter ones against the distortions. In the situation shown in Fig. 10, almost the entire tail is covered by a region of nearly vanishing field strength. 

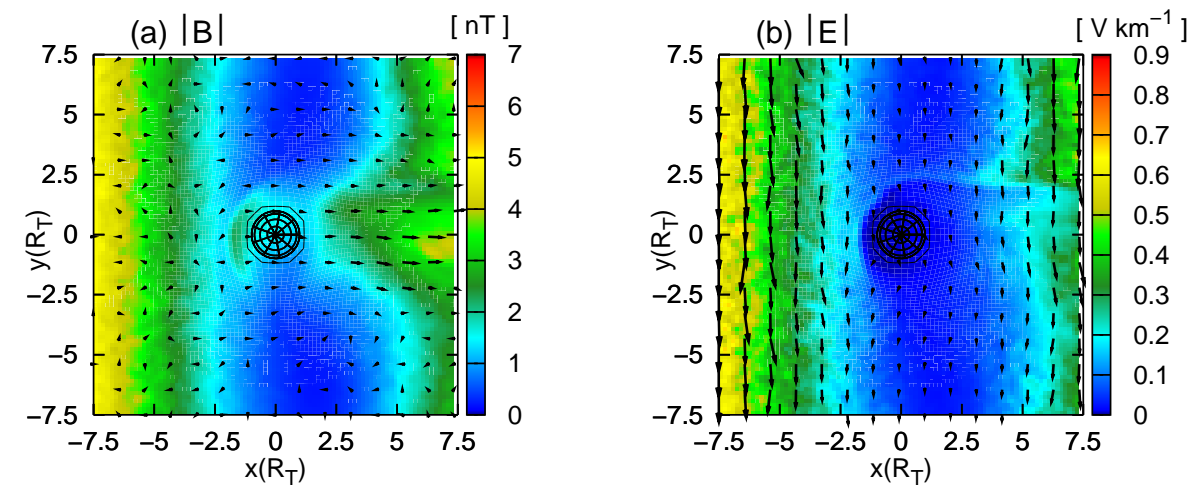

Fig. 9. Run \#4: Titan's plasma environment under the influence of a periodic distortion according to Eq. (12). The period $T=2 \pi / \omega$ of the distortion is $T=3500 \delta t$. The figure shows a snapshot of $E$ and $B$ in the satellite's equatorial plane.
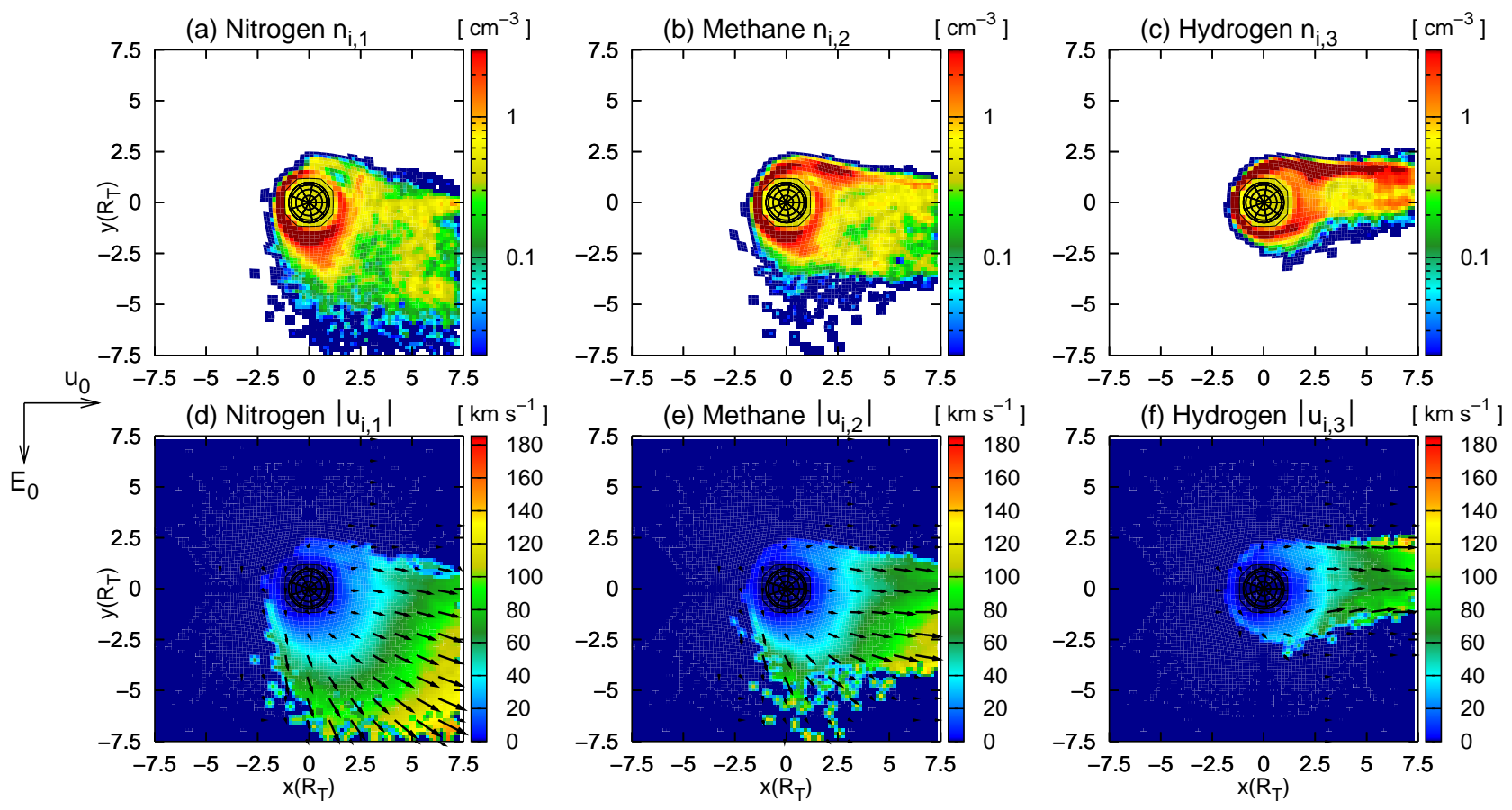

Fig. 10. Run \#4: Titan's plasma environment under the influence of a periodic distortion according to Eq. (12). The period $T=2 \pi / \omega$ of the distortion is $T=3500 \delta t$. The figure shows the ionospheric plasma parameters that correspond to Fig. 9.

However, as can be seen from panels (d) and (e), only the extension of the nitrogen tail in $(y<0)$ direction is significantly larger than in the stationary case (cf. Fig. 2). The $(y<0)$ flank of the major methane concentration is still clearly identifiable and can be found between $y=-2.5 R_{T}$ and $y=-5 R_{T}$, i.e. the methane population is confined to almost the same region as in the stationary case. However, a diffuse "cloud" of methane ions is attached to the the flank of the tail as well (cf. Fig. 10b and e), illustrating that the reduced field strength starts to enlarge the diameter of the methane tail as well. The diameter of the hydrogen tail is practically identical to the stationary case, thus illustrating that the lightest species does not yet experience the reduced field strength. The electric field topology in Fig. 9b illustrates that the decrease of $E$ in the wake does not only emerge from the distortion, but also from the cavity arising from the accumulation of slow ionospheric particles. The flank of this cavity in the $(y>0)$ hemisphere is still identifiable in Fig. 9b. As already illustrated in the foregoing figures, when Titan is exposed to a region of increased field strength, this slow ion population carves 

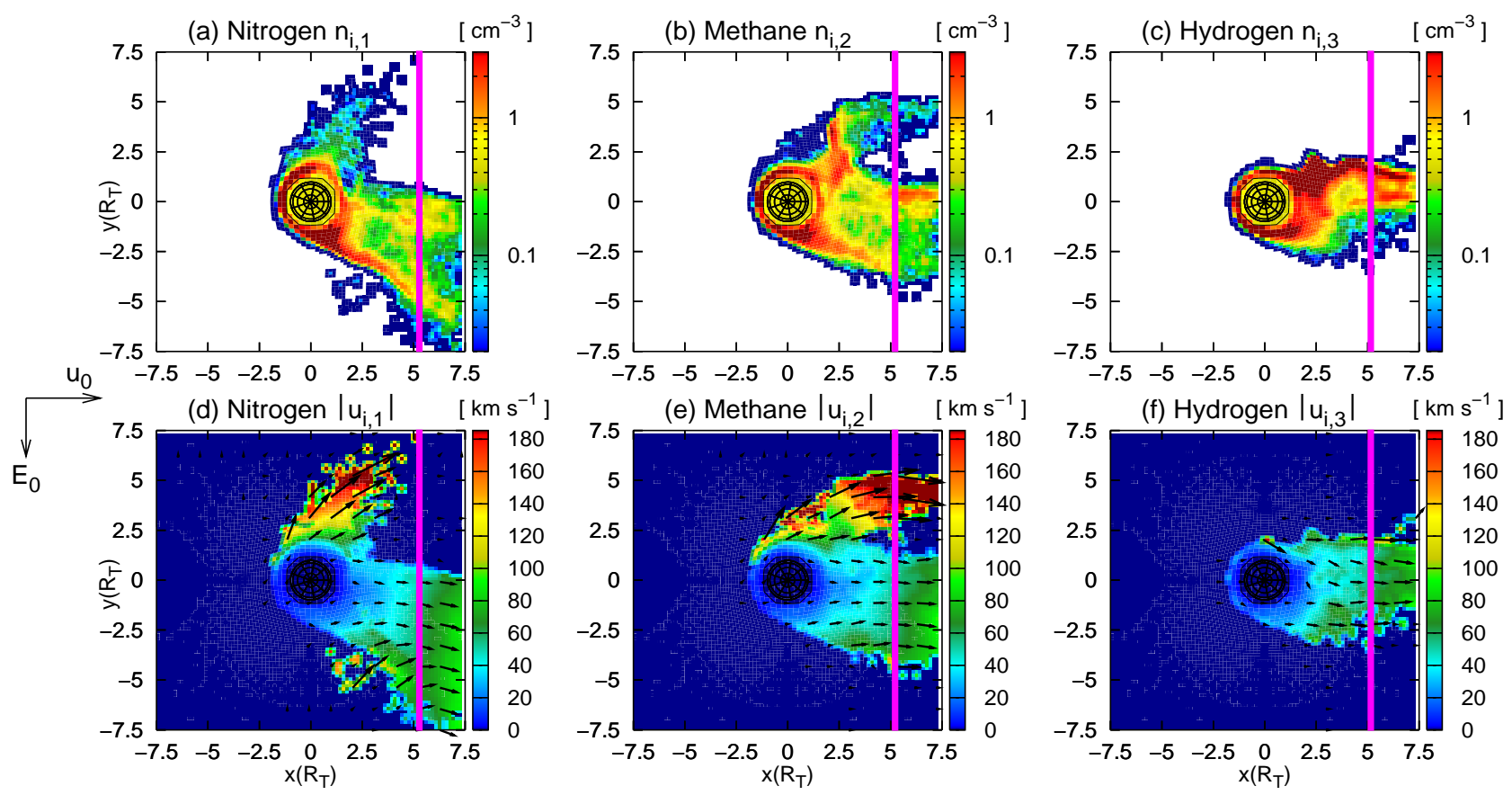

Fig. 11. Run \#5: Influence of a tangential discontinuity on Titan's plasma tail. The discontinuity denotes a reversal of the magnetic field direction from $\boldsymbol{B} \propto-\boldsymbol{e}_{z}$ to $\boldsymbol{B} \propto+\boldsymbol{e}_{z}$. The figure shows the ionospheric plasma parameters when the discontinuity is located at $x=+5 R_{T}$, as denoted by the magenta bars. In other words, in the $\left(x<5 R_{T}\right)$ half space, $\boldsymbol{E}$ points in $(-y)$ direction, while for $\left(x>5 R_{T}\right)$, it is directed in $(+y)$ direction.

some kind of cavity into the distorted magnetospheric fields. When Titan is located in a region of increased $B$ (not shown here), the diameter of the nitrogen tail perpendicular to the flow direction is reduced. The extension of the methane tail is also smaller than in the stationary case, but the difference between both scenarios is only moderate.

The smaller is the period $T$ of the distortion, the shorter are the intervals in which the field magnitude in the vicinity of Titan is nearly constant. When $T$ becomes smaller than a certain critical value, the tail fails to adapt to the changes in the upstream conditions quick enough. In the simulation run with $T=1000 \delta t$, the oscillations in the tail diameters are significantly smaller than in the $T=3500 \delta t$ scenario. The tail extensions show only minor oscillations around an "average" value.

In general, the simulations show that, as long as the ambient electromagnetic fields oscillate only in magnitude while maintaining a constant direction, the structure of the tail does not differ too much from the results for stationary upstream conditions. Under these circumstances, the hemisphere of Titan in which the formation of a pick-up tail is "allowed" is the same during the entire simulation.

\section{$7 \quad$ Influence of field rotations}

In this section, we shall consider a situation in which Ti$\tan$ is exposed to a discontinuous, sudden change in the upstream magnetic field direction. Such a situation may for instance occur when the satellite crosses Saturn's magnetopause in times of high solar wind dynamic pressure and enters the magnetosheath region, as recently observed during T32. In order to study the influence of a discontinuity on Titan's tail structure, we have again considered an extreme situation: After the simulation has been allowed to achieve a quasi-stationary state, the magnetic field in the left-hand inflow boundary layer is reversed, i.e. the field orientation is permanently changed from "downward" to "upward". After that, the field in the boundary layer points again in a constant direction. The new field orientation is convected towards Titan by the magnetospheric plasma flow. In the following, we are interested in the details of the transition that Titan's ionospheric tail undergoes when it encounters this tangential discontinuity. The simulation also allows us to determine a characteristic time scale for the adaption of the tail orientation to the modified upstream field direction.

After the discontinuity has reached Titan, the ionospheric tail orientation changes, i.e. the tail is now shifted into the $(y>0)$ hemisphere. A sequence of snapshots that illustrates how the tail modification proceeds is shown in Figs. 11, 

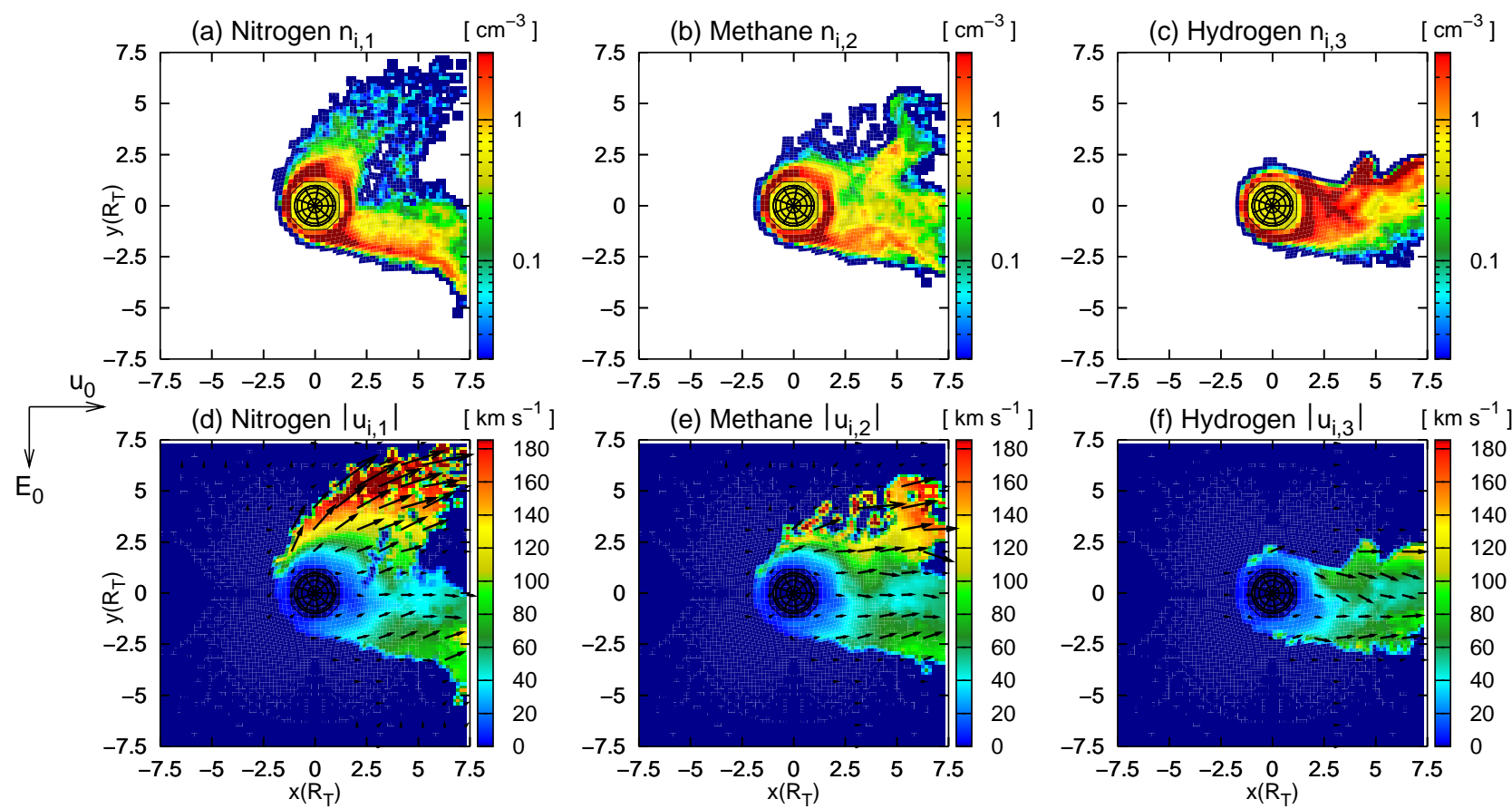

Fig. 12. Run \#5: Influence of a tangential discontinuity on Titan's plasma tail. The discontinuity denotes a reversal of the magnetic field direction from $\boldsymbol{B} \propto-\boldsymbol{e}_{z}$ to $\boldsymbol{B} \propto+\boldsymbol{e}_{z}$. The figure shows the ionospheric plasma parameters when the discontinuity is located at $x=+7.5 R_{T}$, i.e. its location coincides with the right-hand boundary of the simulation domain.

12 and 13. The first important aspect to notice is that the re-orientation of the tail is "continuous", i.e. Titan's ionospheric tail does not experience a complete detachment from the satellite in the analyzed simplified cases. This can only be achieved in the case of very low production rates which would not be realistic for the Titan scenario. The modified orientation of the pick-up fields tends to dismantle the original tail in the $(y<0)$ hemisphere, while a new tail is formed at the $(y>0)$ side of Titan. Figure 11 illustrates the situation right after the beginning of the modification. In analogy to the case of a periodic distortion, the reversed pick-up fields first start to erode the $(y<0)$ flank of the nitrogen tail, while the protected methane tail in the $(y<0)$ half space is not yet affected and is therefore able to maintain the cycloidal shape of its outer flank. Simultaneously, the formation of the "new" methane and nitrogen tails commences in the $(y>0)$ hemisphere. As stated above, in the stationary state, the methane tail is completely covered by the nitrogen-induced electric field cavity, so that - despite their smaller mass - the pick-up of these ions is less efficient than the removal of the nitrogen ions. However, as can be seen in Fig. 11e, during the reorientation of the tail, the methane ions are able to match the high velocity achieved at the outer flank of the nitrogen tail. Since the "new" nitrogen tail has not yet fully developed, the methane ions experience the nearly undisturbed electromagnetic fields in the $(y>0)$ hemisphere and are therefore able to achieve a higher velocity than in the stationary state. The reason for this is the higher inertia of the nitrogen ions. As illustrated in Fig. 11d and e, the development of the new methane tail in the $(y>0)$ hemisphere seems to be "one step ahead" of the nitrogen tail, i.e. the methane ions have already reached the wakeside boundary of the simulation domain. When the nitrogen tail is completed on a much larger time scale, the pick-up of the lighter methane ions in the $(y>0)$ hemisphere is more and more slowed down, as can be seen quite well by following the $(y>0)$ density and velocity patterns of both species through the sequence of snapshots.

The different time scales on which the nitrogen and methane ions are removed from the $(y<0)$ half space are also illustrated in Fig. 12. The removal of the nitrogen tail from the $(y<0)$ hemisphere is nearly complete, while the methane tail has undergone only a minor deformation of its flank. Finally, Fig. 13 shows the situation after the tail re-orientation is nearly complete.

Between the snapshots in Figs. 11 and 13, a total number of only about 500 time steps has passed, which is comparable to the time of 666 steps that the distortion needs to travel from Titan at $x=0$ to the wakeside boundary of the simulation domain.

Finally, the case of a $90^{\circ}$ field rotation shall be briefly discussed. However, since the physical processes occurring in this situation are the same as in the $180^{\circ}$ scenario, no 

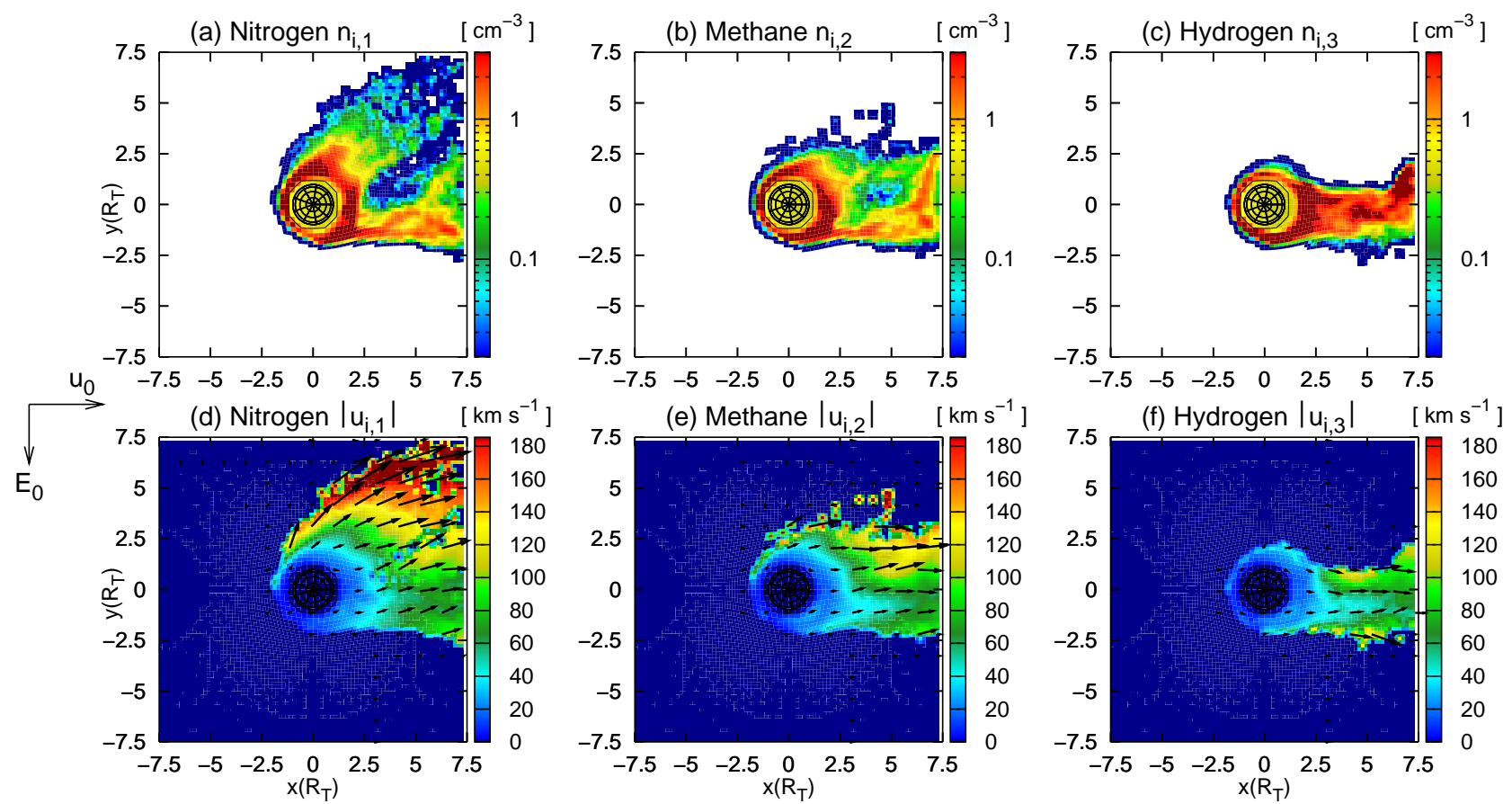

Fig. 13. Run \#5: Influence of a tangential discontinuity on Titan's plasma tail. The discontinuity denotes a reversal of the magnetic field direction from $\boldsymbol{B} \propto-\boldsymbol{e}_{z}$ to $\boldsymbol{B} \propto+\boldsymbol{e}_{z}$. The figure shows the ionospheric plasma parameters about 200 time steps after the discontinuity has left the simulation box. In this time, the undisturbed magnetospheric plasma would travel a distance of $2.3 R_{T}$, i.e. the discontinuity would be located at $x=9.8 R_{T}$.

additional plots are shown here. The tail adjusts to the new direction of the upstream fields, but it is not disconnected from the satellite. Again, the traveling time of the distortion is comparable to the characteristic time scale of the reorientation process. In analogy to the $180^{\circ}$ case, the tails are shifted in sequence, with the nitrogen component being the first one that is affected.

\section{Summary}

Currently, the Cassini mission provides a variety of new scientific information on the interaction between Titan's ionosphere and the impinging magnetospheric plasma of Saturn. In this paper, we present a first attempt to study the influence of non-stationary upstream conditions on Titan's ionospheric tail structure. Since the gyroradii of the particles in Titan's plasma wake are larger than the obstacle itself, a three-dimensional, multispecies hybrid plasma model has been applied.

Three different scenarios have been taken into consideration. In the first one, periodic distortions in the upstream electromagnetic fields have been chosen in such way that a stationary pick-up tail at the anti-Saturn-facing side of Titan is unable to develop. Nonetheless, the simulations show the formation of a central plasma tail behind the obstacle, which is only slightly affected by the distorted ambient electromagnetic fields. These fields do not instantaneously gain access to the entire tail region, but they have to erode the tail from outward to inward. The larger is the period of the distortions, the deeper can the varying fields penetrate into the central plasma tail and the stronger is the orientation of the tail dictated by the ambient magnetospheric plasma conditions. To a certain degree, the heavy ionospheric species are able to shield the lighter ones against the variations in the upstream fields. The results obtained form this scenario may be of value for understanding Titan's plasma interaction in times of highly distorted magnetospheric upstream conditions, e.g. during a substorm.

In the second scenario, the background magnetic field has been modulated by a periodic distortion. In contrast to the first geometry, only the magnitude of the pick-up fields is changed, while they maintain their direction during the entire simulation. The effect of such a distortion on Titan's tail structure is rather weak. The overall orientation of the tail away from Saturn is not changed by the distorted upstream conditions. However, since the field variations affect the gyroradius of the newly generated ionospheric particles, the diameter of the heaviest tail perpendicular to the flow direction is exposed to periodic changes. The periodic deformation of the region populated by $\mathrm{N}_{2}^{+}$ions may be understood as 
a "breathing" of the tail under the influence of the varying electromagnetic fields. Again, the light ionospheric species are only slightly affected by the distortions.

In the third scenario, the influence of a tangential discontinuity on the structure of Titan's plasma wake has been investigated. The results may help to illustrate the transition that Titan's tail undergoes when the satellite crosses Saturn's magnetopause. The simulation has shown that the discontinuity is unable to stop the mass loading process completely and therefore, to detach the tail from the satellite. Instead, the tail adapts to the modified field orientation on a characteristic time scale which is comparable to the time that the magnetospheric flow needs to pass the wake region.

Of course, the nature of the distortions that can be included into the simulations is restricted by the constraints of the numerical model. Incorporating additional variations into the plasma densities and velocities has so far proven impossible, especially due to the finite number of particles in the simulation scenario. Further increasing the time scale of the distortions would require more than 40 passages of the plasma through the simulation domain in order to achieve some kind of quasi-periodic state. Such a simulation can not be handled by any available computer. Therefore, the simulations presented in this paper are meant to illustrate some basic effects that may occur when the magnetospheric plasma conditions near Titan are not stationary, but they will definitely not withstand a direct quantitative comparison to Cassini measurements.

Acknowledgements. The work of S. Simon and U. Motschmann has been supported by the Deutsche Forschungsgemeinschaft through the grant MO 539/15-1. The work of K.-H. Glassmeier was financially supported by the German Ministerium für Wirtschaft und Technologie and by the German Aerospace Center (DLR) under grant $50 \mathrm{OH}$ 9901/4.

Topical Editor I. A. Daglis thanks two anonymous referees for their help in evaluating this paper.

\section{References}

Backes, H.: Titan's Interaction with the Saturnian Magnetospheric Plasma, Ph.D. thesis, Universität zu Köln, 2005.

Backes, H., Neubauer, F. M., Dougherty, M. K., Achilleos, N., André, N., Arridge, C. S., Bertucci, C., Jones, G. H., Khurana, K. K., Russell, C. T., and Wennmacher, A.: Titan's Magnetic Field Signature During the First Cassini Encounter, Science, 308, 992-995, 2005.

Bagdonat, T.: Hybrid Simulation of Weak Comets, Ph.D. thesis, Technische Universität Braunschweig, 2005.

Bagdonat, T. and Motschmann, U.: 3D hybrid simulation of solar wind interaction with comets, in: Space Plasma Simulation Proceedings of the Sixth International School/Symposium ISSS6, edited by: Büchner, J., Dum, C., and Scholer, M., pp. 80-83, 2001.

Bagdonat, T. and Motschmann, U.: 3D Hybrid Simulation Code Using Curvilinear Coordinates, J. Computational Phys., 183, 470$485,2002 \mathrm{a}$.
Bagdonat, T. and Motschmann, U.: From a weak to a strong comet - 3D global hybrid simulation studies, Earth, Moon and Planets, 90, 305-321, 2002b.

Bößwetter, A., Bagdonat, T., Motschmann, U., and Sauer, K.: Plasma boundaries at Mars: A 3D simulation study, Ann. Geophys., 22, 4363-4379, 2004, http://www.ann-geophys.net/22/4363/2004/.

Bößwetter, A., Simon, S., Bagdonat, T., Motschmann, U., Fränz, M., Roussos, E., Krupp, N., Woch, J., Schüle, J., Barabash, S., and Lundin, R.: Comparison of plasma data from ASPERA3/Mars-Express with a 3D hybrid simulation, Ann. Geophys., 25, 1851-1864, 2007, http://www.ann-geophys.net/25/1851/2007/.

Brecht, S. H., Luhmann, J. G., and Larson, D. J.: Simulation of the Saturnian magnetospheric interaction with Titan, J. Geophys. Res., 105, 13 119-13 130, 2000.

Gurnett, D. A., Persoon, A. M., Kurth, W. S., Groene, J. B., Averkamp, T. F., Dougherty, M. K., and Southwood, D. J.: The Variable Rotation Period of the Inner Region of Saturn's Plasma Disk, Science, 316, 442-445, doi:10.1126/science.1138562, 2007.

Kallio, E., Sillanpää, I., and Janhunen, P.: Titan in subsonic and supersonic flow, Geophysical Research Letters, 31, L15 703/1L15 703/4, doi:10.1029/2004GL020344, 2004.

Ledvina, S. A., Luhmann, J. G., Brecht, S. H., and Cravens, T. E.: Titan's induced magnetosphere, Adv. Space Res., 33, 2092 2102, 2004.

Ledvina, S. A., Cravens, T. E., and Kecskemety, K.: Ion distributions in Saturn's magnetosphere near Titan, J. Geophys. Res., 110, 1-16, 2005.

Luhmann, J. G.: Titan's ion exosphere wake: A natural ion mass spectrometer?, J. Geophys. Res., 101, 29387-29393, 1996.

Ma, Y., Nagy, A. F., Cravens, T. E., Sokolov, I. V., Hansen, K. C., Wahlund, J.-E., Crary, F. J., Coates, A. J., and Dougherty, M. K.: Comparisons between MHD model calculations and observations of Cassini flybys of Titan, J. Geophys. Res., 111, A05 207, doi:10.1029/2005JA011 481, 2006.

Ma, Y.-J., Nagy, A. F., Toth, G., Cravens, T. E., Russell, C. T., Gombosi, T. I., Wahlund, J.-E., Crary, F. J., Coates, A. J., Bertucci, C. L., and Neubauer, F. M.: 3D global multi-species Hall-MHD simulation of the Cassini T9 flyby, Geophys. Res. Lett., 34, L24S10, doi:10.1029/2007GL031627, 2007.

Modolo, R., Chanteur, G. M., Wahlund, J.-E., Canu, P., Kurth, W. S., Gurnett, D., Matthews, A. P., and Bertucci, C.: Plasma environment in the wake of Titan from hybrid simulation: A case study, Geophys. Res. Lett., 34, L24S07, doi:10.1029/2007GL030489, 2007a.

Modolo, R., Wahlund, J.-E., Boström, R., Canu, P., Kurth, W. S., Gurnett, D., Lewis, G. R., and Coates, A. J.: Far plasma wake of Titan from the RPWS observations: A case study, Geophys. Res. Lett., 34, L24S04, doi:10.1029/2007GL030482, 2007b.

Motschmann, U. and Kührt, E.: Interaction of the solar wind with weak obstacles: Hybrid simulations for weakly active comets and for Mars, Space Sci. Rev., 122, 197-208, doi:10.1007/s11 214-006-6218-2, 2006.

Neubauer, F. M., Backes, H., Dougherty, M. K., Wennmacher, A., Russell, C. T., Coates, A., Young, D., Achilleos, N., Andre, N., Arridge, C. S., Bertucci, C., Jones, G. H., Khurana, K. K., Knetter, T., Law, A., Lewis, G. R., and Saur, J.: Titan's near mag- 
netotail from magnetic field and plasma observations and modelling: Cassini flybys TA, TB and T3, J. Geophys. Res., 111, A10 220(1-15), doi:10.1029/2006JA011676, 2006.

Neubauer, F. M., Gurnett, D. A., Scudder, J. D., and Hartle, R. E.: Titan's magnetospheric interaction, in: Saturn, edited by: Gehrels, T. and Matthews, M. S., pp. 760-787, University of Arizona Press, Tucson, Arizona, 1984.

Simon, S.: Titan's highly variable plasma environment: A 3D hybrid simulation study, Ph.D. thesis, Technische Universität Braunschweig, 2007.

Simon, S., Bagdonat, T., Motschmann, U., and Glassmeier, K.-H.: Plasma environment of magnetized asteroids: A 3D hybrid simulation study, Ann. Geophys., 24, 407-414, 2006 a.

Simon, S., Boesswetter, A., Bagdonat, T., Motschmann, U., and Glassmeier, K.-H.: Plasma environment of Titan: a 3-D hybrid simulation study, Ann. Geophys., 24, 1113-1135, 2006 b.

Simon, S., Boesswetter, A., Bagdonat, T., and Motschmann, U.: Physics of the Ion Composition Boundary: A comparative 3D hybrid simulation study of Mars and Titan, Ann. Geophys., 25, 99-115, 2007a.

Simon, S., Boesswetter, A., Bagdonat, T., Motschmann, U., and Schuele, J.: Three-dimensional multispecies hybrid simulation of Titan's highly variable plasma environment, Ann. Geophys., 25, 117-144, 2007b.
Simon, S., Kleindienst, G., Boesswetter, A., Bagdonat, T., Motschmann, U., Glassmeier, K.-H., Schuele, J., Bertucci, C., and Dougherty, M. K.: Hybrid simulation of Titan's magnetic field signature during the Cassini T9 flyby, Geophys. Res. Lett., 34, L24S08, doi:10.1029/2007GL029967, 2007.

Simon, S., Motschmann, U., Kleindienst, G., Glassmeier, K.-H., Bertucci, C., and Dougherty, M. K.: Titan's magnetic field signature during the Cassini T34 flyby: Comparison between hybrid simulations and MAG data, Geophys. Res. Lett., in press, 2008.

Snowden, D., Winglee, R., Bertucci, C., and Dougherty, M. K.: Three-dimensional multi-fluid simulation of the plasma interaction at Titan, J. Geophys. Res. (Space Physics), 112(A11), 12 221, doi:10.1029/2007JA012393, 2007.

Wahlund, J.-E., Boström, R., Gustafsson, G., Gurnett, D. A., Kurth, W. S., Pedersen, A., Averkamp, T. F., Hospodarsky, G. B., Persoon, A. M., Canu, P., Neubauer, F. M., Dougherty, M. K., Eriksson, A. I., Morooka, M. W., Gill, R., André, M., Eliasson, L., and Mueller-Wordag, I.: Cassini Measurements of Cold Plasma in the Ionosphere of Titan, Science, 308, 986-989, 2005.

Waite, J. H., Niemann, H., Yelle, R. V., Kasprzak, W. T., Cravens, T. E., Luhmann, J. G., McNutt, R. L., Ip, W.-H., Gell, D., De La Haye, V., Mueller-Wordag, I., Magee, B., Borggren, N., Ledvina, S., Fletcher, G., Walter, E., Miller, R., Scherer, S., Thorpe, R., $\mathrm{Xu}$, J., Block, B., and Arnett, K.: Ion Neutral Mass Spectrometer Results from the First Flyby of Titan, Science, 308, 982-986, 2005. 